\title{
Assessment of Time Delay Threshold Values till Primary Percutaneous Coronary Intervention and their Effects on Coronary Artery Flow, Myocardial Perfusion and Left Ventricular Systolic Function
}

\author{
Dawod Sharif ${ }^{1,2 *}$, Mohamad Watad ${ }^{2}$, Yasmine Sharif ${ }^{2}$, Amal Sharif-Rasslan ${ }^{2,3}$, Uri Rosenschein ${ }^{1,2}$
}

${ }^{1}$ Cardiology Department, Bnai Zion Medical Center, Haifa, Israel.

${ }^{2}$ Technion, Israel Institute of Technology Haifa, Israel.

${ }^{3}$ Department of Mathematics, The Academic Arab College, Haifa, Israel.

dawod.sharif@b-zion.org.il

*Corresponding Author: Dawod Sharif, MD, Department of Cardiology, Bnai Zion Medical Center, 47 Golomb St, Haifa, Israel.

Abstract

Background: Primary percutaneous coronary artery intervention (PCI) in patients with acute ST-elevation myocardial infarction (STEMI) leads to myocardial salvage.

Objectives: To test whether shorter periods to primary PCI improve coronary flow, myocardial perfusion and left ventricular systolic function. Methods: 170 patients with acute anterior STEMI treated by primary PCI were evaluated. Time periods from onset of chest pain to first medical contact (FMC) and from FMC to primary PCI (FMC- PCI) and from pain to primary PCI (Pain - PCI) were recorded.

Results: Patients with pain-PCI<300 min, tended to achieve TIMI grade III more frequently (51\%vs 33\%), $p=0.06$ and more frequent $M B G \geq 2$, (53\% vs 36\%), $p=0.08$. Partial ST-elevation resolution was achieved more frequently in patients with pain- $P C I<180 \mathrm{~min}(87 \%$ vs $65 \%$ ), $p<0.05$. Lower peak troponin levels were found in patients with pain- $P C I<300 \mathrm{~min}(49.2 \pm 45.2 \mathrm{vs} 84.2 \pm 43 \mathrm{ng} / \mathrm{ml}), p=0.03$. In patients with pain- $P C I<180 \mathrm{~min}$, left ventricular ejection fraction (LVEF) increased by $10 \pm 8 \%$ vs $5.5 \pm 5 \%, p<0.05$. In patients with pain-FMC $<90$ and $<120$ min the increase in LVEF was larger than in others, $p<0.05$. FMC- PCI less $<180 \mathrm{~min}$ was associated with larger increase in $L V E F, p<0.05$.

Conclusion: Decreasing pain to PCI intervals, improves myocardial perfusion and LVEF.

Keywords: STEMI; Primary coronary angioplasty; coronary flow; Left ventricular function.

\section{AbBreviations}

PCI: Primary percutaneous coronary artery intervention; STEMI: ST-elevation myocardial infarction; FMC: first medical contact; FMC- PCI: FMC to primary; PCI; Pain - PCI: pain to primary PCI; LVEF: left ventricular ejection fraction; MCI: Mild Cognitive Impairment; AD: Alzheimer's disease; SSRIs: Selective Serotonin Reuptake Inhibitors

\section{INTRODUCTION}

Primary percutaneous coronary intervention (PCI) is the optimal therapy for ST-segment elevation myocardial infarctions (STEMIs) when promptly available (1-5). After occlusion of a coronary artery, the wave front of necrosis progresses from the center of necrosis in the subendocardium, towards the epicardial and adjacent ischemic but still viable regions (6). Rescue of these peri-necrotic viable but ischemic regions, must be performed promptly, since the time from hospital arrival to primary PCI-the door-to-balloon time-is associated with morbidity and mortality (7-8). Door-to-balloon time became a quality measure of hospital performance, and thus, North American and European guidelines recommend a door-to-balloon time of $\leq 90$ minutes (9-12). 
Assessment of Time Delay Threshold Values till Primary Percutaneous Coronary Intervention and their Effects on Coronary Artery Flow, Myocardial Perfusion and Left Ventricular Systolic Function

Coronary artery flow (13) and left ventricular systolic function (14) after primary PCI are important predictors of prognosis. Coronary artery flow is very important in predicting recovery of left ventricular systolic function in patients with acute STEMI treated by primary PCI (15-16). Coronary artery and myocardial flow after primary PCI are dynamic and reflect the dynamic nature of the coronary microcirculation after primary PCI (17).

The present study was performed to test the hypothesis that time delays after the onset of the chest pain of STEMI and hospital arrival till primary PCI affect coronary artery flow, myocardial perfusion and left ventricular systolic function and to find significant time thresholds.

\section{Materials ANd Methods}

One hundred and seventy consecutive patients with acute anterior STEMI undergoing PPCI were enrolled in the study. All fulfilled the following criteria: 1) First anterior wall STEMI. 2) Primary PCI within 12 hours of the onset of symptoms. 3) Routine informed consent to perform primary PCI. Anterior STEMI was defined as continuous chest pain for at least 30 minutes and ST elevation of at least $2.0 \mathrm{~mm}$ in $\geq 2$ contiguous precordial ECG leads. Exclusion criteria included one of the following clinical or angiographic findings: Prior bypass surgery, previous anterior STEMI, significant left main artery disease, failed primary PCI. Pain to first medical contact (FMC), FMC to PCI and pain to PCI time intervals (18) were registered for all patients during the study period from the beginning 2007 to the end of 2013.

Primary PCI was performed in standard fashion. All subjects were treated with either loading oral clopidogrel (600 $\mathrm{mg}$ ) and aspirin (300 $\mathrm{mg}$ ) in the emergency department and maintenance dose of clopidogrel $75 \mathrm{mg} /$ day and aspirin $100 \mathrm{mg}$ /day or with prasugrel loading $60 \mathrm{mg}$ orally and maintained later on $10 \mathrm{mg} /$ day with the same regimen of oral aspirin. Anticoagulation before was achieved with heparin bolus 50-70 units/kg body weight to achieve coagulation time $\geq 250 \mathrm{msec}$ or with bivalirudin bolus $0.75 \mathrm{mg} / \mathrm{kg}$ body weight and maintenance dose of $1.75 \mathrm{mg} / \mathrm{kg} /$ hour intra-venously for 4 hours after the procedure. Coronary angiography and PPCI were performed subsequently. Bare metal stents were deployed by high-pressure implantation techniques. Low magnification angiogram at either the right

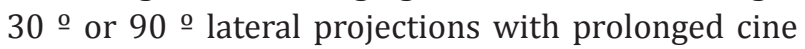
was performed to optimize myocardial blush grade (MBG) documentation at the end of the intervention as previously described (19). All patients were treated with clopidogrel or prasugrel and aspirin for 12 months after the procedure.

Fourteen-lead electrocardiograms were performed at admission and after primary PCI. Measurement of STsegment in mm was performed from the lead of highest elevation. The ratio of difference of ST-elevation before and after primary PCI to ST elevation before primary PCI was calculated. When the ratio exceeded 70\% STelevation resolution was considered complete, a ratio between $30 \%$ and $70 \%$ was considered partial, and no resolution of ST elevation was diagnosed when the ratio was less than $30 \%$.

All patients had routine clinical evaluation and determination of Killip class. Routine blood tests and assessment of troponin and creatine phosphokinase blood levels were measured daily.

All patients had complete Doppler echcardiographic studies, within the first 6 hours after primary PCI, 48 hours later, and 5 days after the intervention. Siemens, Acuson Sequoia echocardiographic system, California, equipped with 3.5-7MHZ transducers was used. All patients had complete Doppler echcordiographic studies, within the first 6 hours after primary PCI, 48 hours after PPCI, and 5 days after primary PCI. Chamber diameters and usual measurements were performed according to recommendations of American Society of Echocardiography. Ejection fraction of LV (LVEF) was measured from biplane apical views.

For the calculation of wall motion score index

$$
L V-W M S I=\frac{\sum \text { scoreof } 16 \text { segments }}{16}
$$

assigning a value of 1 for normal $\mathrm{LV}$ wall motion, 2 for hypokinesis and 3 for akinesis. Using the same values of wall motion scores, LAD 9 segmental score index was calculated as:

$$
L A D-W M S I=\frac{\sum \text { scoreof } 9 \text { segments }}{9}
$$

In order to obtain LAD flows, the color Doppler Nyquist limit was set at $17 \mathrm{~cm} / \mathrm{sec}$. From low parasternal short axis view, search for diastolic color flow in the anterior 
Assessment of Time Delay Threshold Values till Primary Percutaneous Coronary Intervention and their Effects on Coronary Artery Flow, Myocardial Perfusion and Left Ventricular Systolic Function

interventricular groove followed by clockwise rotation was performed, while form apical foreshortened two chamber views LAD diastolic flow was located in the interventricular groove and the counterclockwise rotation of the transducer was performed.

Parameters of LAD velocity patterns were averaged from 3 beats, all in sinus rhythm. Diastolic LAD deceleration Time (DDT) was measured as the time from peak diastolic velocity to the intercept of tangent of the velocity envelope with baseline.

\section{STATistical ANAlysis}

Statistical analysis was conducted using SPSS software version 16. Continuous parameters were expressed as means and standard deviations, and categorical variables were expressed by numbers or percentage. Two-tailed student's-t test was performed to compare changes in continuous parameters. Categorical variables were compared by the $\chi 2$ test. $\mathrm{p}<0.05$ was considered as statistically significant.

\section{RESULTS}

One hundred and seventy patients with acute anterior STEMI treated by primary PCI, age $61 \pm 12.7$ years, were evaluated. Most of the patients were men and the clinical characteristics are summarized in table 1.

\section{Variation of Time Intervals}

After 2010, FMC to PCI time decreased significantly, and caused a reduction in Pain to PCI time despite the fact that Pain to FMC did not change, table 2.

\section{Effects of Pain to PCI Time Intervals}

Angiographic Findings: Post primary PCI, patients with pain to $\mathrm{PCI}<300 \mathrm{~min}$, tended to achieve TIMI grade III more frequently (51\%) compared to others (33\%), $\mathrm{p}=0.06$ and more frequent $\mathrm{MBG} \geq 2,(53 \%$ vs $36 \%), p=0.08$.

ST-Elevation Resolution: Partial ST-elevation resolution after $\mathrm{PCI}$, was achieved more frequently in patients with pain to $\mathrm{PCI}<180 \mathrm{~min}(87 \%$ vs $65 \%)$, $\mathrm{p}<0.05$, table 4 .

LAD Velocity Parameters: Early assessment after PCI revealed lower LAD systolic velocities in patients with pain to PCI less than $300 \mathrm{~min}$, tables 5. Patients with pain to PCI intervals less than 90 and less than $120 \mathrm{~min}$ had lower LAD diastolic time velocity integrals compared to others. Patients with pain to PCI intervals less than $180 \mathrm{~min}$ had shorter LAD diastolic deceleration time table 5 .

Late after PCI, lower diastolic velocities and integrals were found in those with pain to PCI less than 90min, table 6. Systolic LAD velocities were lower in those with pain to PCI less than 300 min.

Myocardial Damage: Lower peak troponin blood levels were found in patients with pain to $\mathrm{PCI}<300$ $\min (49.2 \pm 45.2$ vs $84.2 \pm 43 \mathrm{ng} / \mathrm{ml}), \mathrm{p}=0.03$, table 7 .

Left Ventricular Systolic Function: Patients with pain to PCI intervals less than $180 \mathrm{~min}$ and less than $230 \mathrm{~min}$ had larger increase in LVEF before discharge, table 8 .

\section{Effects of Components of Pain to PCI Time Intervals}

Angiographic Findings: TIMI and myocardial blush grades after PCI were not affected by pain to FMC and FMC to PCI intervals, table 3.

ST-Elevation Resolution: Complete and partial ST elevation resolution was not affected by pain to FMC; while FMC to PCI intervals less than 90 min were associated with higher prevalence of partial STelevation resolution, table 4 .

LAD Velocity Parameters: Early LAD systolic velocity was lower in those with pain to FMC less than 180 min, table 5 . In patients with FMC to PCI intervals less than 90 and $135 \mathrm{~min}$ had lower early LAD systolic velocities and integrals, table 5. Late diastolic LAD velocities were lower in those with pain to FMC less than $90 \mathrm{~min}$, table 6 . While late LAD systolic velocities were lower in those with FMC to PCI less than $180 \mathrm{~min}$, the late diastolic deceleration and pressure half times were longer, table 6 .

Myocardial Damage: Maximal blood levels of myocardial biomarkers were not affected neither by pain to FMC nor by FMC to PCI intervals, table 7.

Left Ventricular Systolic Function: In patients with pain to FMC less than 90 and less than 120 min, the increase in LVEF was larger than in others, $\mathrm{p}<0.05$. FMC to PCI less than $180 \mathrm{~min}$ was associated with larger increase in LVEF, $\mathrm{p}<0.05$, table 8. 
Assessment of Time Delay Threshold Values till Primary Percutaneous Coronary Intervention and their Effects on Coronary Artery Flow, Myocardial Perfusion and Left Ventricular Systolic Function

Table 1. Patients' Characteristics.

\begin{tabular}{|l|c|}
\hline Characteristics & Patients \\
\hline Patients (number) & 170 \\
\hline Women (\%) & $25.9 \%$ \\
\hline Age (yrs) & $61 \pm 12.7$ \\
\hline HTN (\%) & $47 \%$ \\
\hline Obesity (\%) & $30.6 \%$ \\
\hline FH of CAD (\%) & $27.7 \%$ \\
\hline HLP (\%) & $56.5 \%$ \\
\hline DM2 (\%) & $18.2 \%$ \\
\hline Smoker (\%) & $49.4 \%$ \\
\hline Killip I class (\%) & $92.4 \%$ \\
\hline Pain to PCI & $219.7 \pm 155.7$ \\
\hline
\end{tabular}

Abbreviations: HTN=Hypertension; HLP= Hyperlipidemia; DM2= Diabetes Mellitus type 2; FH of CAD= Family history of coronary artery disease.

Table 2. Change overtime of time intervals till primary percutaneous coronary intervention.

\begin{tabular}{|l|l|l|l|}
\hline & Pain to FMC & FMC to PCI & Pain to PCI \\
\hline Before 2010 & $150.1 \pm 177.7$ & $98.3 \pm 124.8$ & $\mathbf{2 2 5 . 0 1} \pm \mathbf{1 6 7 . 5}$ \\
\hline After 2010 & $131.5 \pm 127.4$ & $75.6 \pm 46.1$ & $\mathbf{1 8 8 . 6} \pm 98.6$ \\
\hline P-value & 0.332 & 0.037 & $\mathbf{0 . 0 3 0 4}$ \\
\hline
\end{tabular}

Abbreviations: FMC= First Medical Contact.

Table 3. Angiographic coronary flow and myocardial blush

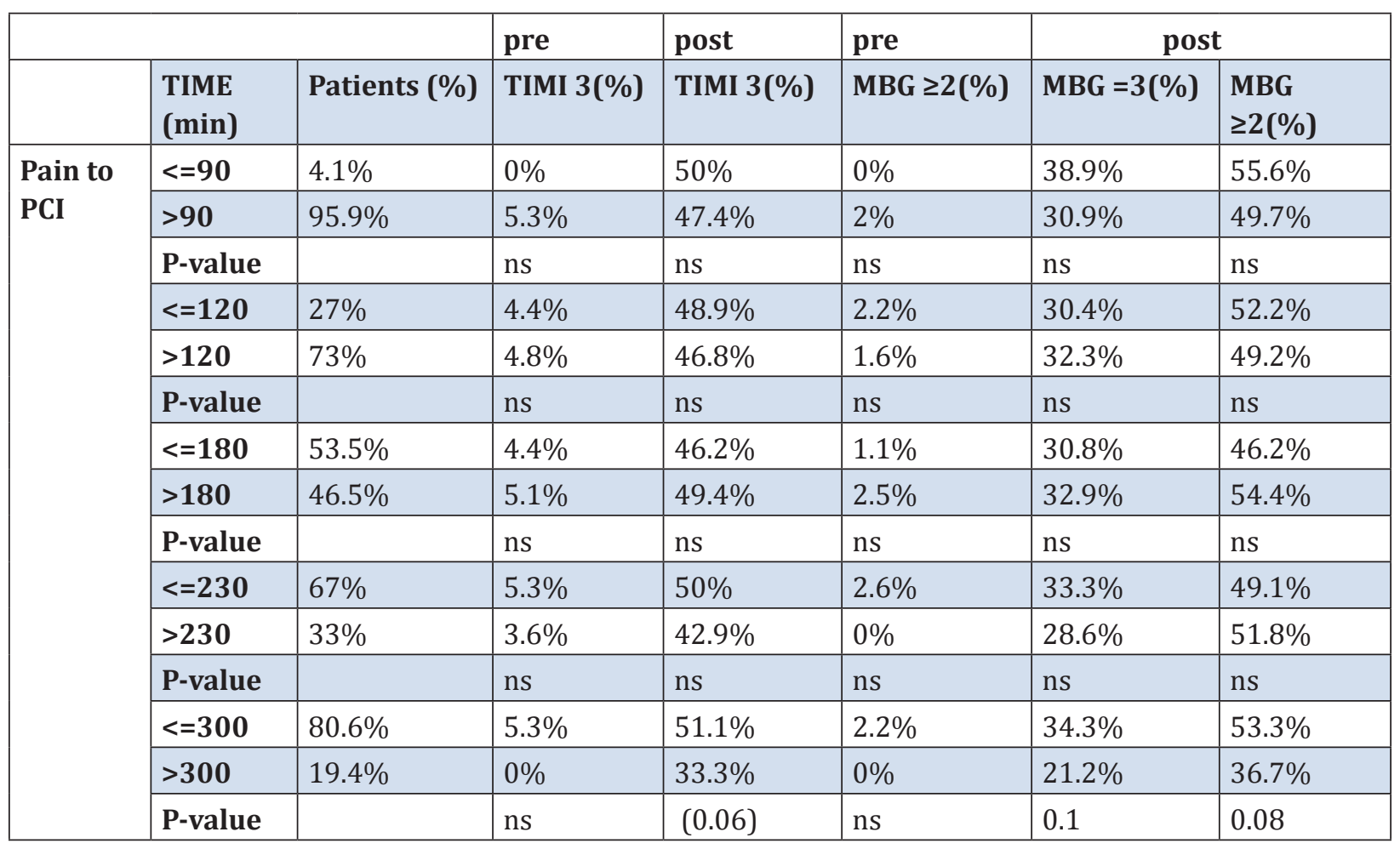


Assessment of Time Delay Threshold Values till Primary Percutaneous Coronary Intervention and their Effects on Coronary Artery Flow, Myocardial Perfusion and Left Ventricular Systolic Function

\begin{tabular}{|c|c|c|c|c|c|c|c|}
\hline \multirow{12}{*}{\begin{tabular}{|l} 
Pain to \\
FMC
\end{tabular}} & $<=60$ & $45 \%$ & $3.4 \%$ & $95.2 \%$ & $1.7 \%$ & $71.4 \%$ & $90.5 \%$ \\
\hline & $>60$ & $55 \%$ & $8.2 \%$ & $90 \%$ & $2.7 \%$ & $57.4 \%$ & $97.1 \%$ \\
\hline & p-value & & ns & ns & ns & ns & ns \\
\hline & $<=90$ & $68 \%$ & $4.1 \%$ & $93.3 \%$ & $1.02 \%$ & $55.6 \%$ & $91.1 \%$ \\
\hline & $>90$ & $32 \%$ & $8.7 \%$ & $88.6 \%$ & $4.3 \%$ & $64 \%$ & $97 \%$ \\
\hline & p-value & & ns & ns & ns & ns & ns \\
\hline & $<=120$ & $73 \%$ & $4.03 \%$ & $91.5 \%$ & $1.6 \%$ & $59.3 \%$ & $91.5 \%$ \\
\hline & $>120$ & $27 \%$ & $17.4 \%$ & $90 \%$ & $1.2 \%$ & $63.3 \%$ & $96.7 \%$ \\
\hline & p-value & & $<0.05$ & ns & ns & ns & ns \\
\hline & $<=180$ & $88 \%$ & $5.3 \%$ & $91.03 \%$ & $2 \%$ & $59 \%$ & $93.6 \%$ \\
\hline & $>180$ & $12 \%$ & $0 \%$ & $90.9 \%$ & $0 \%$ & $72.7 \%$ & $90.9 \%$ \\
\hline & p-value & & ns & ns & ns & ns & ns \\
\hline \multirow{12}{*}{$\begin{array}{l}\text { FMC to } \\
\text { PCI }\end{array}$} & $<=45$ & $24 \%$ & $4.9 \%$ & $88 \%$ & $2.4 \%$ & $41.5 \%$ & 93.6\% \\
\hline & $>45$ & $76 \%$ & $6.3 \%$ & $91.1 \%$ & $1.5 \%$ & $28.5 \%$ & $94.7 \%$ \\
\hline & p-value & & ns & ns & ns & ns & ns \\
\hline & $<=90$ & $63.5 \%$ & $51.9 \%$ & $2.8 \%$ & $36.1 \%$ & $3.7 \%$ & $14.8 \%$ \\
\hline & $>90$ & $36.5 \%$ & $40.3 \%$ & $0 \%$ & $24.2 \%$ & $4.8 \%$ & $12.9 \%$ \\
\hline & p-value & & ns & ns & ns & ns & ns \\
\hline & $<=135$ & $74.1 \%$ & $8.8 \%$ & $2.4 \%$ & $4.7 \%$ & $4.2 \%$ & $14.3 \%$ \\
\hline & $>135$ & $25.9 \%$ & $9.3 \%$ & $0 \%$ & $5.9 \%$ & $4.8 \%$ & $4.8 \%$ \\
\hline & p-value & & ns & ns & ns & ns & ns \\
\hline & $<=180$ & $84.1 \%$ & $90.5 \%$ & $2.1 \%$ & $3.1 \%$ & $16.1 \%$ & $4.2 \%$ \\
\hline & $>180$ & $15.9 \%$ & $100 \%$ & $0 \%$ & $3.7 \%$ & $3.7 \%$ & $4.8 \%$ \\
\hline & p-value & & ns & ns & ns & ns & ns \\
\hline
\end{tabular}

Abbreviations: FMC= First Medical Contact; Time (min)= pain to FMC time (minutes)/Door to Balloon/Pain to Balloon; Pre TIMI= TIMI grade prior to PPCI; Post TIMI= TIMI grade after PPCI. TIMI3 post\%=percentage of patients with TIMI grade flow 3 after PPCI; Pre MBG= Myocardial blush grade prior to PPCI; Post MBG= Myocardial blush grade after PPCI; MBG 3 Post $\%=$ percentage of patients having MBG=3 post $P P C I ; M B G \geq 2$ post $\%$ =Percentage of patients having $\mathrm{MBG} \geq 2$ post $\mathrm{PPCI}$. ns= non significant.

Table 4. ST-Elevation resolution

\begin{tabular}{|c|c|c|c|c|c|c|}
\hline & $\begin{array}{l}\text { TIME } \\
\text { (min) }\end{array}$ & Patients(\%) & $\begin{array}{l}\text { Complete } \\
\text { Resolution } \\
\geq 70 \%\end{array}$ & $\begin{array}{l}\text { Partial } \\
\text { Resolution } \\
\text { 30\%-70\% }\end{array}$ & $\begin{array}{l}\text { No } \\
\text { resolution < } \\
30 \%\end{array}$ & $\begin{array}{l}\text { Resolution } \\
\text { Average }\end{array}$ \\
\hline \multirow{15}{*}{$\begin{array}{l}\text { Pain to } \\
\text { PCI }\end{array}$} & $<=90$ & $4.1 \%$ & $22 \%$ & $77.8 \%$ & $11 \%$ & $50 \% \pm 32.03 \%$ \\
\hline & $>90$ & $95.9 \%$ & $15.8 \%$ & $53.9 \%$ & $19.7 \%$ & $48 \% \pm 29.8 \%$ \\
\hline & $p$-value & & ns & ns & ns & ns \\
\hline & $<=120$ & $27 \%$ & $16.7 \%$ & $83.9 \%$ & $12.5 \%$ & $45.4 \% \pm 29.2 \%$ \\
\hline & $>120$ & $73 \%$ & $26.2 \%$ & $73.8 \%$ & $21.3 \%$ & $49.3 \% \pm 30.3 \%$ \\
\hline & $p$-value & & ns & $\mathrm{ns}$ & ns & ns \\
\hline & $<=180$ & $53.5 \%$ & $13.3 \%$ & $86.7 \%$ & $15.6 \%$ & $45.1 \% \pm 27.1 \%$ \\
\hline & $>180$ & $46.5 \%$ & $35 \%$ & $65 \%$ & $22.5 \%$ & $51.17 \% \pm 32.7 \%$ \\
\hline & $p$-value & & $<0.05$ & $<0.05$ & ns & ns \\
\hline & $<=230$ & $67 \%$ & $22.4 \%$ & $77.6 \%$ & $17.3 \%$ & $48.4 \% \pm 29.6 \%$ \\
\hline & $>230$ & $33 \%$ & $25.9 \%$ & $74.1 \%$ & $22.2 \%$ & $47.8 \% \pm 31 \%$ \\
\hline & $\mathrm{p}$-value & & ns & ns & ns & ns \\
\hline & $<=300$ & $80.6 \%$ & $24.7 \%$ & $75.3 \%$ & $17.8 \%$ & $48.8 \% \pm 30 \%$ \\
\hline & $>300$ & $19.4 \%$ & $16.7 \%$ & $83.3 \%$ & $25 \%$ & $44.7 \% \pm 29.9 \%$ \\
\hline & $p$-value & & ns & ns & ns & ns \\
\hline
\end{tabular}


Assessment of Time Delay Threshold Values till Primary Percutaneous Coronary Intervention and their Effects on Coronary Artery Flow, Myocardial Perfusion and Left Ventricular Systolic Function

\begin{tabular}{|c|c|c|c|c|c|c|}
\hline \multirow{12}{*}{\begin{tabular}{|l} 
Pain to \\
FMC
\end{tabular}} & $<=60$ & $45 \%$ & $35 \%$ & $60 \%$ & $5 \%$ & $56.7 \% \pm 28.2 \%$ \\
\hline & $>60$ & $55 \%$ & $20 \%$ & $56.9 \%$ & $23.1 \%$ & $45.6 \% \pm 30.1 \%$ \\
\hline & $\mathrm{p}$-value & & ns & ns & $\mathrm{ns}(0.07)$ & ns \\
\hline & $<=90$ & $68 \%$ & $26.8 \%$ & $63.4 \%$ & $9.8 \%$ & $51 \% \pm 28.2 \%$ \\
\hline & $>90$ & $32 \%$ & $20.4 \%$ & $52.3 \%$ & $27.3 \%$ & $45.6 \% \pm 31.4 \%$ \\
\hline & p-value & & ns & ns & $0.039<0.05$ & ns \\
\hline & $<=120$ & $73 \%$ & $23.2 \%$ & $58.9 \%$ & $17.9 \%$ & $48.1 \% \pm 30.1 \%$ \\
\hline & $>120$ & $27 \%$ & $24.1 \%$ & $55.2 \%$ & $20.7 \%$ & $48.3 \% \pm 29.9 \%$ \\
\hline & p-value & & ns & ns & ns & ns \\
\hline & $<=180$ & $88 \%$ & $24.6 \%$ & $56.2 \%$ & $19.2 \%$ & $48.5 \% \pm 30.5 \%$ \\
\hline & $>180$ & $12 \%$ & $16.7 \%$ & $66.6 \%$ & $16.7 \%$ & $46.4 \% \pm 26.9 \%$ \\
\hline & p-value & & ns & ns & ns & ns \\
\hline \multirow{12}{*}{$\begin{array}{l}\text { FMC to } \\
\text { PCI }\end{array}$} & $<=45$ & $24 \%$ & $18.8 \%$ & $59.3 \%$ & $21.9 \%$ & $43.4 \% \pm 30.9 \%$ \\
\hline & $>45$ & $76 \%$ & $26.4 \%$ & $56.6 \%$ & $17 \%$ & $51.1 \% \pm 29.2 \%$ \\
\hline & p-value & & ns & ns & ns & ns \\
\hline & $<=90$ & $63.5 \%$ & $17.2 \%$ & $64 \%$ & $18.8 \%$ & $46 \% \pm 28.2 \%$ \\
\hline & $>90$ & $36.5 \%$ & $42.8 \%$ & $38.1 \%$ & $19.1 \%$ & $54.8 \% \pm 34.4 \%$ \\
\hline & $\mathrm{p}$-value & & $<0.05$ & $<0.05$ & ns & ns \\
\hline & $<=135$ & $74.1 \%$ & $18.7 \%$ & $61.3 \%$ & $20 \%$ & $46.1 \pm 29.2 \%$ \\
\hline & $>135$ & $25.9 \%$ & $60 \%$ & $30 \%$ & $50 \%$ & $63.4 \% \pm 31.7 \%$ \\
\hline & p-value & & $<0.05$ & ns & $<0.05$ & ns \\
\hline & $<=180$ & $84.1 \%$ & $23.2 \%$ & $58.5 \%$ & $18.3 \%$ & $48.4 \% \pm 29.8 \%$ \\
\hline & $>180$ & $15.9 \%$ & $33.4 \%$ & $33.3 \%$ & $33.3 \%$ & $41.7 \% \pm 38.2 \%$ \\
\hline & $\mathrm{p}$-value & & ns & ns & ns & ns \\
\hline
\end{tabular}

Abbreviations: FMC= First Medical Contact; Time (min)= pain to FMC time (minutes); ns= non significant.

Table 5. Early LAD Doppler velocity parameters.

\begin{tabular}{|c|c|c|c|c|c|c|c|c|c|c|c|}
\hline & $\begin{array}{|lll|}\text { T I } & \text { M } & \text { E } \\
(\min )\end{array}$ & \begin{tabular}{|l|} 
Patients \\
$(\%)$
\end{tabular} & $\begin{array}{l}\text { L A D } \\
\text { Diam } \\
\text { (mm) }\end{array}$ & \begin{tabular}{|l|} 
VD \\
(cm / \\
sec)
\end{tabular} & $\begin{array}{l}\text { VS } \\
(\mathrm{cm} / \mathrm{sec})\end{array}$ & TVI D (cm) & $\begin{array}{l}\text { TVIS } \\
\text { (cm) }\end{array}$ & $\begin{array}{l}\text { PHT } \\
\text { (msec) }\end{array}$ & \begin{tabular}{|l|} 
DDT \\
(msec)
\end{tabular} & \begin{tabular}{|l|} 
DDT $>600$ \\
$(\mathrm{msec})(\%)$
\end{tabular} & \begin{tabular}{|l|} 
HR \\
(bpm)
\end{tabular} \\
\hline \multirow[t]{10}{*}{$\begin{array}{l}\text { Pain to } \\
\text { PCI }\end{array}$} & $<=90$ & $4.1 \%$ & $\begin{array}{l}2 \pm \\
1.4\end{array}$ & $\begin{array}{l}36.9 \pm \\
11.7\end{array}$ & $\begin{array}{l}6.9 \pm \\
16\end{array}$ & $9.28 \pm 2.8$ & $\begin{array}{l}2.23 \pm \\
2.6\end{array}$ & $\begin{array}{l}167 \pm \\
170.3\end{array}$ & $\begin{array}{l}351.7 \pm \\
234.3\end{array}$ & 25 & $\begin{array}{l}72.5 \pm \\
6.9\end{array}$ \\
\hline & $>90$ & $95.9 \%$ & \begin{tabular}{|l|}
$2.52 \pm$ \\
0.44 \\
\end{tabular} & \begin{tabular}{|l}
$42.9 \pm$ \\
16.2 \\
\end{tabular} & $\begin{array}{l}13.4 \pm \\
11.4 \\
\end{array}$ & $12.02 \pm 5.1$ & $\begin{array}{l}2.79 \pm \\
2.12\end{array}$ & $\begin{array}{l}162.3 \pm \\
96.3\end{array}$ & $\begin{array}{l}563.6 \pm \\
330.3 \\
\end{array}$ & 47.4 & $76.7 \pm 9.6$ \\
\hline & p-value & & ns & ns & ns & $0.038<0.05$ & ns & ns & 0.044 & ns & ns \\
\hline & $<=120$ & $27 \%$ & \begin{tabular}{|l|}
$2.57 \pm$ \\
0.68 \\
\end{tabular} & \begin{tabular}{|l}
$39.6 \pm$ \\
14.5 \\
\end{tabular} & $\begin{array}{l}10.9 \pm \\
13.2\end{array}$ & $10.4 \pm 4.03$ & \begin{tabular}{|l}
$2.48 \pm$ \\
2.2
\end{tabular} & $\begin{array}{l}153.1 \pm \\
135.2\end{array}$ & \begin{tabular}{|l|}
$447.1 \pm$ \\
313.7 \\
\end{tabular} & 28.6 & $75.1 \pm 9.3$ \\
\hline & $>120$ & $73 \%$ & \begin{tabular}{|l|}
$2.45 \pm$ \\
0.41 \\
\end{tabular} & \begin{tabular}{|l|}
$43.2 \pm$ \\
16.3 \\
\end{tabular} & $\begin{array}{l}13.3 \pm \\
11.7 \\
\end{array}$ & $12.2 \pm 5.3$ & \begin{tabular}{|l|}
$2.83 \pm$ \\
2.2 \\
\end{tabular} & $\begin{array}{l}167.7 \pm \\
93.8\end{array}$ & \begin{tabular}{|l|}
$580.6 \pm$ \\
326.8 \\
\end{tabular} & 52.3 & $\begin{array}{l}76.5 \pm \\
9.4\end{array}$ \\
\hline & $p$-value & & ns & ns & ns & $<0.05$ & ns & ns & ns & ns & ns \\
\hline & $<=180$ & $53.5 \%$ & \begin{tabular}{|l|}
$2.44 \pm$ \\
0.6 \\
\end{tabular} & \begin{tabular}{|l}
43.03 \\
\pm 16.1 \\
\end{tabular} & $\begin{array}{l}10.1 \pm \\
15.34\end{array}$ & $11.1 \pm 5.2$ & \begin{tabular}{|l|}
$2.48 \pm$ \\
2.62 \\
\end{tabular} & $\begin{array}{l}146.5 \pm \\
123.4 \\
\end{array}$ & \begin{tabular}{|l|}
$454.4 \pm$ \\
329.6 \\
\end{tabular} & 30.3 & $\begin{array}{l}76.07 \pm \\
9.02\end{array}$ \\
\hline & $>180$ & $46.5 \%$ & \begin{tabular}{|l|}
$2.53 \pm$ \\
0.41
\end{tabular} & $\begin{array}{l}40.9 \pm \\
15.35\end{array}$ & $\begin{array}{l}15.2 \pm \\
6.03\end{array}$ & $12.2 \pm 4.6$ & $\begin{array}{l}2.98 \pm \\
1.5\end{array}$ & $\begin{array}{l}182.04 \pm \\
84.7\end{array}$ & $\begin{array}{l}623.1 \pm \\
304.3\end{array}$ & 59.38 & $\begin{array}{l}76 \pm \\
9.8\end{array}$ \\
\hline & p-value & & ns & ns & ns & ns & ns & ns & 0.035 & $0.018<0.05$ & ns \\
\hline & $<=230$ & $67 \%$ & $\begin{array}{l}2.5 \pm \\
0.56\end{array}$ & $\begin{array}{l}42.2 \pm \\
14.6\end{array}$ & $\begin{array}{l}12 \pm \\
13.7\end{array}$ & $11.6 \pm 4.9$ & $\begin{array}{l}2.7 \pm \\
2.34\end{array}$ & $\begin{array}{l}160.7 \pm \\
118\end{array}$ & $\begin{array}{l}525.4 \pm \\
342.9\end{array}$ & 42.2 & $74.9 \pm 9.3$ \\
\hline
\end{tabular}


Assessment of Time Delay Threshold Values till Primary Percutaneous Coronary Intervention and their Effects on Coronary Artery Flow, Myocardial Perfusion and Left Ventricular Systolic Function

\begin{tabular}{|c|c|c|c|c|c|c|c|c|c|c|c|}
\hline & $>230$ & $33 \%$ & \begin{tabular}{|l|}
$2.47 \pm$ \\
0.9 \\
\end{tabular} & \begin{tabular}{|l|}
$41.7 \pm$ \\
18.4 \\
\end{tabular} & $\begin{array}{l}13.86 \pm \\
7.5 \\
\end{array}$ & $11.7 \pm 5.08$ & \begin{tabular}{|l|}
$2.78 \pm$ \\
1.65 \\
\end{tabular} & $\begin{array}{l}169.2 \pm \\
75.9 \\
\end{array}$ & $\begin{array}{l}564.7 \pm \\
291.5 \\
\end{array}$ & 50 & $79.6 \pm 8.7$ \\
\hline & p-value & & ns & ns & ns & ns & ns & ns & ns & ns & ns \\
\hline & $<=300$ & $80.6 \%$ & \begin{tabular}{|l|}
$2.5 \pm$ \\
0.51
\end{tabular} & \begin{tabular}{|l}
$40.9 \pm$ \\
15 \\
\end{tabular} & $\begin{array}{l}11.9 \pm \\
12.6 \\
\end{array}$ & $11.5 \pm 5.04$ & \begin{tabular}{|l|}
$2.69 \pm$ \\
2.2 \\
\end{tabular} & $\begin{array}{l}166.5 \pm \\
110.8 \\
\end{array}$ & $\begin{array}{l}552.2 \pm \\
333.5 \\
\end{array}$ & 45.8 & $75.6 \pm 8.9$ \\
\hline & $>300$ & $19.4 \%$ & \begin{tabular}{|l|}
$2.3 \pm$ \\
0.56
\end{tabular} & \begin{tabular}{|l}
$51.3 \pm$ \\
19.6 \\
\end{tabular} & $\begin{array}{l}17.8 \pm \\
4.48 \\
\end{array}$ & $12.7 \pm 4.09$ & $\begin{array}{l}3 \pm \\
1.56 \\
\end{array}$ & $\begin{array}{l}127 \pm \\
67.1\end{array}$ & $\begin{array}{l}392.3 \pm \\
227.8\end{array}$ & 33.3 & $81.75 \pm 3.2$ \\
\hline & p-value & & ns & ns & $0.032<0.05$ & ns & ns & ns & ns & ns & ns \\
\hline \multirow[t]{12}{*}{$\begin{array}{l}\text { Pain to } \\
\text { FMC }\end{array}$} & $<=60$ & $45 \%$ & $\begin{array}{l}2.48 \pm \\
0.43\end{array}$ & $\begin{array}{l}42.3 \pm \\
16.1\end{array}$ & $\begin{array}{l}16.1 \pm \\
6.8\end{array}$ & $11.5 \pm 4.4$ & $\begin{array}{l}3.46 \pm \\
1.78\end{array}$ & $\begin{array}{l}160 \pm \\
140.1\end{array}$ & $\begin{array}{l}487.1 \pm \\
323.5\end{array}$ & 33.3 & $\begin{array}{l}72.2 \pm \\
10.5\end{array}$ \\
\hline & $>60$ & $55 \%$ & \begin{tabular}{|l|}
$2.49 \pm$ \\
0.54 \\
\end{tabular} & \begin{tabular}{|l|}
$42 \pm$ \\
15.7 \\
\end{tabular} & $\begin{array}{l}11.4 \pm \\
13.2 \\
\end{array}$ & $\begin{array}{l}11.7 \pm \\
5.16 \\
\end{array}$ & $\begin{array}{l}2.48 \pm \\
2.23 \\
\end{array}$ & \begin{tabular}{|l|}
$164 \pm$ \\
96.7 \\
\end{tabular} & $\begin{array}{l}552.6 \pm \\
328.7 \\
\end{array}$ & 48 & \begin{tabular}{|l|}
$77.5 \pm$ \\
8.5 \\
\end{tabular} \\
\hline & p-value & & ns & ns & ns & ns & ns & ns & ns & ns & 0.09 \\
\hline & $<=90$ & $68 \%$ & \begin{tabular}{|l|}
$2.45 \pm$ \\
0.65 \\
\end{tabular} & \begin{tabular}{|l}
$40.7 \pm$ \\
13.5 \\
\end{tabular} & \begin{tabular}{|l|}
$12.4 \pm$ \\
11.5 \\
\end{tabular} & $11.25 \pm 4.6$ & $\begin{array}{l}2.63 \pm \\
2 \\
\end{array}$ & \begin{tabular}{|l|}
$151.3 \pm$ \\
1219 \\
\end{tabular} & \begin{tabular}{|l}
$484.9 \pm$ \\
317.7 \\
\end{tabular} & 35.5 & \begin{tabular}{|l}
$73.8 \pm$ \\
9.5 \\
\end{tabular} \\
\hline & $>90$ & $32 \%$ & \begin{tabular}{|l|}
$2.52 \pm$ \\
0.32 \\
\end{tabular} & \begin{tabular}{|l|}
$43.4 \pm$ \\
17.6 \\
\end{tabular} & \begin{tabular}{|l|}
$12.7 \pm$ \\
12.9 \\
\end{tabular} & $12.04 \pm 5.3$ & \begin{tabular}{|l}
$2.8 \pm$ \\
2.3 \\
\end{tabular} & \begin{tabular}{|l|}
$173.9 \pm$ \\
93.5 \\
\end{tabular} & \begin{tabular}{|l|}
$586.2 \pm$ \\
331.3 \\
\end{tabular} & 52.9 & $78.6 \pm 8.5$ \\
\hline & p-value & & ns & ns & ns & ns & ns & ns & ns & ns & 0.057 \\
\hline & $<=120$ & $73 \%$ & \begin{tabular}{|l|}
$2.48 \pm$ \\
0.57 \\
\end{tabular} & \begin{tabular}{|l}
$42.6 \pm$ \\
15.3 \\
\end{tabular} & $\begin{array}{l}11.6 \pm \\
13.6 \\
\end{array}$ & $\begin{array}{l}11.3 \pm \\
4.6\end{array}$ & $\begin{array}{l}2.61 \pm \\
2.3 \\
\end{array}$ & $\begin{array}{l}151.1 \pm \\
115.5\end{array}$ & $\begin{array}{l}318.7 \pm \\
288.8\end{array}$ & 36.4 & $75.5 \pm 9.3$ \\
\hline & $>120$ & $27 \%$ & \begin{tabular}{|l|}
$2.49 \pm$ \\
0.34 \\
\end{tabular} & \begin{tabular}{|l}
$40.8 \pm$ \\
16.9 \\
\end{tabular} & $\begin{array}{l}14.5 \pm \\
8 \\
\end{array}$ & $12.5 \pm 5.7$ & $\begin{array}{l}2.97 \pm \\
1.8 \\
\end{array}$ & \begin{tabular}{|l|}
$188.2 \pm$ \\
86.8 \\
\end{tabular} & \begin{tabular}{|l|}
$648.4 \pm$ \\
320.6 \\
\end{tabular} & 61.9 & $77.3 \pm 9.4$ \\
\hline & p-value & & ns & ns & ns & ns & ns & $<0.05$ & 0.06 & ns & ns \\
\hline & $<=180$ & $88 \%$ & \begin{tabular}{|l|}
$2.47 \pm$ \\
0.52 \\
\end{tabular} & \begin{tabular}{|l|}
$41.4 \pm$ \\
15.3 \\
\end{tabular} & $\begin{array}{l}11.8 \pm \\
12.7 \\
\end{array}$ & $11.4 \pm 4.9$ & \begin{tabular}{|l}
$2.61 \pm$ \\
2.2 \\
\end{tabular} & \begin{tabular}{|l|}
$162.6 \pm$ \\
113.2 \\
\end{tabular} & \begin{tabular}{|l}
$534.5 \pm$ \\
339.4 \\
\end{tabular} & 42.9 & $75.6 \pm 9.03$ \\
\hline & $>180$ & $12 \%$ & $\begin{array}{|lr|}2 . & 7 \pm \\
0.4 & \\
\end{array}$ & \begin{tabular}{|l}
$47.2 \pm$ \\
18.7 \\
\end{tabular} & $\begin{array}{l}18.03 \pm \\
3.9 \\
\end{array}$ & $14.2 \pm 5.35$ & \begin{tabular}{|l}
$3.7 \pm$ \\
0.99 \\
\end{tabular} & $\begin{array}{l}166.2 \pm \\
45.9 \\
\end{array}$ & $\begin{array}{l}567 \pm \\
156.2 \\
\end{array}$ & 66.7 & \begin{tabular}{|l}
$80.6 \pm$ \\
11.7 \\
\end{tabular} \\
\hline & p-value & & ns & ns & $0.0175<0.05$ & ns & ns & ns & ns & ns & ns \\
\hline \multirow[t]{12}{*}{$\begin{array}{l}\text { FMC to } \\
\text { PCI }\end{array}$} & $<=45$ & $24 \%$ & \begin{tabular}{|l|}
$2.42 \pm$ \\
0.72 \\
\end{tabular} & \begin{tabular}{|l|}
$44.4 \pm$ \\
16.7 \\
\end{tabular} & $\begin{array}{l}6.6 \pm \\
16.6 \\
\end{array}$ & $\begin{array}{llll}1 & 1.8 & 5 & \pm \\
5.86 & & & \\
\end{array}$ & $\begin{array}{l}1.8 \pm \\
2.7 \\
\end{array}$ & $\begin{array}{l}143.9 \pm \\
105.1 \\
\end{array}$ & $\begin{array}{l}506.3 \pm \\
357.8 \\
\end{array}$ & 40 & \begin{tabular}{|l|}
$77.8 \pm$ \\
8.72 \\
\end{tabular} \\
\hline & $>45$ & $76 \%$ & $\begin{array}{l}2.5 \pm \\
0.55 \\
\end{array}$ & \begin{tabular}{|l|}
$40.7 \pm$ \\
15.1 \\
\end{tabular} & $\begin{array}{l}15.7 \pm \\
7.3 \\
\end{array}$ & $11.5 \pm 4.4$ & \begin{tabular}{|l}
$3.2 \pm$ \\
1.6 \\
\end{tabular} & \begin{tabular}{|l|}
$175.4 \pm$ \\
109.2 \\
\end{tabular} & \begin{tabular}{|l}
$577 \pm$ \\
307.9 \\
\end{tabular} & 47.5 & $74.8 \pm 9.9$ \\
\hline & p-value & & ns & ns & 0.033 & ns & 0.039 & ns & ns & ns & ns \\
\hline & $<=90$ & $63.5 \%$ & \begin{tabular}{|l|}
$2.5 \pm$ \\
0.52 \\
\end{tabular} & \begin{tabular}{|l|}
$41 \pm$ \\
16 \\
\end{tabular} & $\begin{array}{l}1 \pm \\
13.4 \\
\end{array}$ & $11.2 \pm 5.04$ & \begin{tabular}{|l|}
$2.98 \pm$ \\
2.3 \\
\end{tabular} & \begin{tabular}{|l|}
$159.8 \pm$ \\
115.9 \\
\end{tabular} & \begin{tabular}{|l|}
$521.6 \pm$ \\
344.5 \\
\end{tabular} & 42 & $76.6 \pm 9.1$ \\
\hline & $>90$ & $36.5 \%$ & \begin{tabular}{|l|}
$2.56 \pm$ \\
0.42 \\
\end{tabular} & \begin{tabular}{|l|}
$45.5 \pm$ \\
14.6 \\
\end{tabular} & \begin{tabular}{|l|}
$17.4 \pm$ \\
4.1 \\
\end{tabular} & $13.1 \pm 4.4$ & \begin{tabular}{|l|}
$3.47 \pm$ \\
1.45 \\
\end{tabular} & \begin{tabular}{|l|}
$174.9 \pm$ \\
75.9 \\
\end{tabular} & \begin{tabular}{|l|}
$598.2 \pm$ \\
259.2 \\
\end{tabular} & 53.3 & $74.4 \pm 10.2$ \\
\hline & p-value & & ns & ns & $0.0103<0.05$ & ns & ns & ns & ns & ns & ns \\
\hline & $<=135$ & $74.1 \%$ & \begin{tabular}{|l|}
$2.5 \pm$ \\
0.52
\end{tabular} & \begin{tabular}{|l}
$41.1 \pm$ \\
15.3 \\
\end{tabular} & $\begin{array}{l}11.8 \pm \\
12.7 \\
\end{array}$ & $11.5 \pm 5.03$ & $\begin{array}{l}2.6 \pm \\
2.2\end{array}$ & $\begin{array}{l}162.4 \pm \\
109.3 \\
\end{array}$ & $\begin{array}{l}530.5 \pm \\
326.9\end{array}$ & 43.9 & 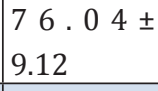 \\
\hline & $>135$ & $25.9 \%$ & \begin{tabular}{|l|}
$2.32 \pm$ \\
0.39 \\
\end{tabular} & \begin{tabular}{|l|}
$48.7 \pm$ \\
17.9 \\
\end{tabular} & $\begin{array}{l}17.4 \pm \\
5.03 \\
\end{array}$ & $12.8 \pm 4.4$ & $\begin{array}{l}3.4 \pm \\
1.9 \\
\end{array}$ & $\begin{array}{l}167.8 \pm \\
102.9\end{array}$ & \begin{tabular}{|l}
$587.4 \pm$ \\
338.2
\end{tabular} & 50 & $76.2 \pm 11.2$ \\
\hline & p-value & & ns & ns & $0.04<0.05$ & ns & ns & ns & ns & ns & ns \\
\hline & $<=180$ & $84.1 \%$ & \begin{tabular}{|l|}
$2.5 \pm$ \\
0.49 \\
\end{tabular} & \begin{tabular}{|l}
$41.1 \pm$ \\
15 \\
\end{tabular} & \begin{tabular}{|l|}
$12.4 \pm$ \\
12.3 \\
\end{tabular} & $\begin{array}{l}11.6 \pm \\
5 \\
\end{array}$ & $\begin{array}{l}2.77 \pm \\
2.17 \\
\end{array}$ & \begin{tabular}{|l|}
$165.4 \pm$ \\
107.3 \\
\end{tabular} & \begin{tabular}{|l|}
$549.8 \pm$ \\
323.7 \\
\end{tabular} & 46.03 & $75.8 \pm 9.2$ \\
\hline & $>180$ & $15.9 \%$ & \begin{tabular}{|l|}
$1.7 \pm$ \\
0.02 \\
\end{tabular} & $\begin{array}{l}65 \pm \\
21.2 \\
\end{array}$ & $\begin{array}{l}15 \pm \\
4.2 \\
\end{array}$ & $12.75 \pm 6.2$ & $\begin{array}{l}1.4 \pm \\
0.42 \\
\end{array}$ & \begin{tabular}{|l}
$33 \pm$ \\
0.4 \\
\end{tabular} & $\begin{array}{l}150 \pm \\
50.9 \\
\end{array}$ & 0 & \begin{tabular}{|l}
$88 \pm$ \\
9 \\
\end{tabular} \\
\hline & p-value & & $<0.05$ & ns & ns & ns & 0.034 & $<0.05$ & 0.0007 & ns & ns \\
\hline
\end{tabular}

Abbreviations: FMC= First Medical Contact; Time (min)= pain to FMC time (minutes); VD=Peak diastolic velocity; VS=Peak systolic velocity; TVI D= Diastolic time velocity integral; TVI S=Systolic time velocity integral; PHT=Pressure half time; DDT=Diastolic deceleration time; bmp: Beat per minute; ns= non significant. 
Assessment of Time Delay Threshold Values till Primary Percutaneous Coronary Intervention and their Effects on Coronary Artery Flow, Myocardial Perfusion and Left Ventricular Systolic Function

Table 6. Late LAD Doppler velocity parameters

\begin{tabular}{|c|c|c|c|c|c|c|c|c|c|c|c|}
\hline & $\begin{array}{l}\text { T I M E } \\
\text { (min) }\end{array}$ & $\begin{array}{l}\text { Patients } \\
(\%)\end{array}$ & $\begin{array}{l}\text { L A } \quad \text { D } \\
\text { D i a m } \\
(\mathrm{mm})\end{array}$ & \begin{tabular}{|l} 
VD \\
$(\mathrm{cm} / \mathrm{sec})$
\end{tabular} & $\begin{array}{l}\text { VS } \\
(\mathrm{cm} / \mathrm{sec})\end{array}$ & TVI D (cm) & $\begin{array}{l}\text { TVI S } \\
(\mathrm{cm})\end{array}$ & \begin{tabular}{|l} 
PHT \\
(msec)
\end{tabular} & \begin{tabular}{|l} 
DDT \\
(msec)
\end{tabular} & $\begin{array}{l}\text { DDT > } \\
600 \\
(\mathrm{msec}) \\
(\%)\end{array}$ & \begin{tabular}{|l} 
HR \\
$(\mathrm{bpm})$
\end{tabular} \\
\hline \multirow[t]{15}{*}{\begin{tabular}{|l} 
Pain to \\
PCI
\end{tabular}} & $<=90$ & $4.1 \%$ & \begin{tabular}{|l|}
$1.8 \pm$ \\
1.13
\end{tabular} & $\begin{array}{l}30.8 \pm \\
7.2\end{array}$ & $\begin{array}{l}0.75 \pm \\
19.24\end{array}$ & $\begin{array}{l}9.12 \pm \\
3.48\end{array}$ & $1.2 \pm 2.74$ & $\begin{array}{l}197.4 \pm \\
155.5\end{array}$ & $\begin{array}{l}672.8 \pm \\
535.9\end{array}$ & 60 & $\begin{array}{ll}78.4 & \pm \\
19.8 & \end{array}$ \\
\hline & $>90$ & $95.9 \%$ & \begin{tabular}{|l|}
$3.26 \pm$ \\
3.19 \\
\end{tabular} & \begin{tabular}{|l}
$40.3 \pm$ \\
13.9
\end{tabular} & $\begin{array}{l}15.96 \pm \\
8.9\end{array}$ & $\begin{array}{l}13.9 \pm \\
4.3\end{array}$ & $3.67 \pm 2.4$ & $\begin{array}{l}202.9 \pm \\
78.8\end{array}$ & $\begin{array}{l}654.8 \pm \\
258.8\end{array}$ & 64.3 & $\begin{array}{l}69.9 \pm \\
11.5\end{array}$ \\
\hline & $\mathbf{P}$ & & ns & $0.037<0.05$ & ns & $0.033<0.05$ & ns & ns & ns & ns & ns \\
\hline & $<=120$ & $27 \%$ & \begin{tabular}{|l|}
$2.56 \pm$ \\
0.61
\end{tabular} & $\begin{array}{l}36.15 \pm \\
8.66\end{array}$ & $\begin{array}{l}13 \pm \\
13.9\end{array}$ & $\begin{array}{l}12.3 \pm \\
2.76\end{array}$ & $\begin{array}{l}2.88 \pm \\
2.38\end{array}$ & $\begin{array}{l}193.1 \pm \\
95.1\end{array}$ & $\begin{array}{l}657.95 \\
\pm 326.5\end{array}$ & 70 & $\begin{array}{l}72.45 \pm \\
14.5\end{array}$ \\
\hline & $>120$ & $73 \%$ & $\begin{array}{l}3.46 \pm \\
3.73\end{array}$ & $\begin{array}{l}41.17 \pm \\
15.4\end{array}$ & $\begin{array}{l}15.7 \pm \\
8.7\end{array}$ & $\begin{array}{l}14.1 \pm \\
4.95\end{array}$ & $3.76 \pm 2.5$ & $\begin{array}{l}207.2 \pm \\
81.4\end{array}$ & $\begin{array}{l}655.5 \pm \\
265\end{array}$ & 61 & $\begin{array}{l}70.03 \pm \\
11.9\end{array}$ \\
\hline & $\mathbf{p}$ & & ns & ns & ns & ns & ns & ns & ns & ns & ns \\
\hline & $<=180$ & $53.5 \%$ & \begin{tabular}{|l|}
$2.63 \pm$ \\
0.54
\end{tabular} & \begin{tabular}{|l|}
$37.8 \pm$ \\
13.3 \\
\end{tabular} & $\begin{array}{l}13.2 \pm \\
13.27\end{array}$ & $\begin{array}{l}12.6 \pm \\
3.9\end{array}$ & $\begin{array}{l}2.98 \pm \\
2.48\end{array}$ & $\begin{array}{l}196.97 \\
\pm 88.1\end{array}$ & $\begin{array}{l}674.1 \pm \\
300.9\end{array}$ & 68.8 & $\begin{array}{l}71.5 \quad \pm \\
14.23\end{array}$ \\
\hline & $>180$ & $46.5 \%$ & \begin{tabular}{|l|}
$3.83 \pm$ \\
4.6 \\
\end{tabular} & \begin{tabular}{|l}
$41.7 \pm$ \\
14.1 \\
\end{tabular} & $\begin{array}{l}16.54 \pm \\
6.5 \\
\end{array}$ & $\begin{array}{l}14.7 \pm \\
4.9 \\
\end{array}$ & \begin{tabular}{|lll}
4.0 & $1 \pm$ \\
2.43 & & \\
\end{tabular} & \begin{tabular}{|l}
$208.8 \pm$ \\
84.01 \\
\end{tabular} & \begin{tabular}{|l|}
$636.7 \pm$ \\
267.7 \\
\end{tabular} & 58.6 & $\begin{array}{l}70.3 \pm \\
11 \\
\end{array}$ \\
\hline & p & & ns & ns & ns & ns & ns & ns & ns & ns & ns \\
\hline & $<=230$ & $67 \%$ & \begin{tabular}{|l|}
$2.61 \pm$ \\
0.51
\end{tabular} & $\begin{array}{l}37.95 \pm \\
12.3\end{array}$ & $\begin{array}{l}13.8 \pm \\
11.61 \\
\end{array}$ & $\begin{array}{l}12.82 \pm \\
3.8 \\
\end{array}$ & $3.34 \pm 2.7$ & $\begin{array}{l}196.3 \pm \\
84\end{array}$ & $\begin{array}{l}667.3 \pm \\
283.5\end{array}$ & 65.9 & \begin{tabular}{|l|}
$70.88 \pm$ \\
13.5 \\
\end{tabular} \\
\hline & $>230$ & $33 \%$ & \begin{tabular}{|l}
$4.33 \pm$ \\
5.3
\end{tabular} & $\begin{array}{l}43.2 \pm \\
16.2 \\
\end{array}$ & $\begin{array}{l}17 \pm \\
7.62\end{array}$ & $\begin{array}{l}15.1 \pm \\
5.4 \\
\end{array}$ & $\begin{array}{l}3.79 \pm \\
1.99\end{array}$ & $\begin{array}{l}215.2 \pm \\
90.2\end{array}$ & $\begin{array}{l}633.8 \pm \\
290.6\end{array}$ & 60 & $\begin{array}{l}71.3 \pm \\
11.6\end{array}$ \\
\hline & $\mathbf{p}$ & & ns & ns & ns & ns & ns & ns & ns & ns & ns \\
\hline & $<=300$ & $80.6 \%$ & $\begin{array}{l}3.24 \pm \\
3.4\end{array}$ & $\begin{array}{l}38 \pm \\
12.56\end{array}$ & $\begin{array}{l}13.89 \pm \\
10.67\end{array}$ & $\begin{array}{l}13.04 \pm \\
4.2\end{array}$ & $\begin{array}{l}3.33 \pm \\
2.44\end{array}$ & $\begin{array}{l}208.5 \pm \\
87.2\end{array}$ & $\begin{array}{l}675.6 \pm \\
291.7\end{array}$ & 66.04 & $\begin{array}{l}70.73 \pm \\
12.9\end{array}$ \\
\hline & $>300$ & $19.4 \%$ & \begin{tabular}{|l}
$2.88 \pm$ \\
0.38 \\
\end{tabular} & $\begin{array}{l}50 \pm \\
17.2\end{array}$ & $\begin{array}{l}20.75 \pm \\
7,17\end{array}$ & $17.2 \pm 4.98$ & $\begin{array}{l}4.49 \pm \\
2.69\end{array}$ & $\begin{array}{l}156.7 \pm \\
60.5\end{array}$ & $\begin{array}{l}528.25 \\
\pm 192.7 \\
\end{array}$ & 50 & $\begin{array}{l}72.7 \pm \\
13.6\end{array}$ \\
\hline & p & & ns & 0.09 & $0.038<0.05$ & ns & ns & ns & ns & ns & ns \\
\hline \multirow[t]{12}{*}{$\begin{array}{l}\text { Pain to } \\
\text { FMC }\end{array}$} & $<=60$ & $45 \%$ & \begin{tabular}{|l|}
$2.7 \pm$ \\
0.37
\end{tabular} & $\begin{array}{l}35.6 \pm \\
8.1\end{array}$ & $\begin{array}{l}16.7 \pm \\
5.3\end{array}$ & $\begin{array}{l}13 \pm \\
4.2\end{array}$ & \begin{tabular}{|l}
$3.9 \pm$ \\
1.6 \\
\end{tabular} & $\begin{array}{l}194.9 \pm \\
99.9\end{array}$ & $\begin{array}{l}664.4 \pm \\
344.7\end{array}$ & 64.3 & $\begin{array}{l}69.2 \pm \\
12.03\end{array}$ \\
\hline & $>60$ & $55 \%$ & \begin{tabular}{|l}
$3.3 \pm$ \\
0.35
\end{tabular} & $\begin{array}{l}40.7 \pm \\
14.8 \\
\end{array}$ & $\begin{array}{l}14.3 \pm \\
11.6 \\
\end{array}$ & $\begin{array}{l}13.7 \pm \\
4.5 \\
\end{array}$ & \begin{tabular}{|l|}
$3.4 \pm$ \\
2.7 \\
\end{tabular} & $\begin{array}{l}204.7 \pm \\
81.9 \\
\end{array}$ & $\begin{array}{l}653.9 \pm \\
267.4\end{array}$ & 63.8 & $\begin{array}{l}71.5 \pm \\
13.3 \\
\end{array}$ \\
\hline & p & & ns & ns & ns & ns & ns & ns & ns & ns & ns \\
\hline & $<=90$ & $68 \%$ & \begin{tabular}{|l|}
$2.62 \pm$ \\
0.6 \\
\end{tabular} & $\begin{array}{l}35.6 \pm \\
9.1 \\
\end{array}$ & $\begin{array}{l}13.5 \pm \\
11.8 \\
\end{array}$ & $\begin{array}{l}12.4 \pm \\
3.8 \\
\end{array}$ & \begin{tabular}{|l|}
$3.4 \pm$ \\
2.9 \\
\end{tabular} & $\begin{array}{l}187.03 \pm \\
85.9\end{array}$ & $\begin{array}{l}631 \pm \\
291.7 \\
\end{array}$ & 60 & $\begin{array}{l}70.1 \pm \\
10.6 \\
\end{array}$ \\
\hline & $>90$ & $32 \%$ & $\begin{array}{l}3.6 \pm \\
0.41\end{array}$ & \begin{tabular}{|l|}
$43.2 \pm$ \\
16.2 \\
\end{tabular} & $\begin{array}{l}16.1 \pm \\
9.25 \\
\end{array}$ & $\begin{array}{l}14.6 \pm \\
4.8 \\
\end{array}$ & \begin{tabular}{|l}
$3.6 \pm$ \\
2.1 \\
\end{tabular} & $\begin{array}{l}217.2 \pm \\
84.3 \\
\end{array}$ & $\begin{array}{l}680.8 \pm \\
278.7\end{array}$ & 67.7 & $\begin{array}{l}72.0 \pm \\
15.2 \\
\end{array}$ \\
\hline & $\mathbf{p}$ & & ns & $0.026<0.05$ & ns & ns & ns & ns & ns & ns & ns \\
\hline & $<=120$ & $73 \%$ & $\begin{array}{l}2.6 \pm \\
0.5\end{array}$ & $\begin{array}{l}39.2 \pm \\
14.4 \\
\end{array}$ & $\begin{array}{l}14.1 \pm \\
12.1 \\
\end{array}$ & $\begin{array}{l}12.9 \pm \\
4.16\end{array}$ & $\begin{array}{l}3.4 \pm \\
2.8\end{array}$ & $\begin{array}{l}188.8 \pm \\
84.4\end{array}$ & $\begin{array}{l}640.6 \pm \\
286.5\end{array}$ & 62.5 & $\begin{array}{l}75.1 \pm \\
11.6\end{array}$ \\
\hline & $>120$ & $27 \%$ & $\begin{array}{l}4.4 \pm \\
5.5\end{array}$ & $\begin{array}{l}40.3 \pm \\
12.4 \\
\end{array}$ & $\begin{array}{l}16.25 \pm \\
6.6\end{array}$ & $\begin{array}{l}14.9 \pm \\
4.8 \\
\end{array}$ & \begin{tabular}{|l}
$3.6 \pm$ \\
1.9 \\
\end{tabular} & $\begin{array}{l}228.8 \pm \\
84.2\end{array}$ & $\begin{array}{l}686.2 \pm \\
283.2\end{array}$ & 66.7 & $\begin{array}{l}70.7 \pm \\
15.8\end{array}$ \\
\hline & p & & ns & ns & ns & ns & ns & ns & ns & ns & ns \\
\hline & $<=180$ & $88 \%$ & $\begin{array}{l}3.2 \pm \\
3.3\end{array}$ & $\begin{array}{c}38.7 \pm \\
13.4\end{array}$ & $\begin{array}{l}14.3 \pm \\
10.6\end{array}$ & $\begin{array}{l}13.1 \pm \\
3.9\end{array}$ & $\begin{array}{l}3.4 \pm \\
2.5\end{array}$ & $\begin{array}{l}206.2 \pm \\
88.6\end{array}$ & $\begin{array}{l}671.9 \pm \\
293.6\end{array}$ & 66.04 & $\begin{array}{l}70.8 \pm \\
11.4\end{array}$ \\
\hline & $>180$ & $12 \%$ & \begin{tabular}{|lr}
3 & \pm \\
0.41 &
\end{tabular} & $\begin{array}{l}45.5 \pm \\
15.4 \\
\end{array}$ & $\begin{array}{l}18.5 \pm \\
9.2 \\
\end{array}$ & $\begin{array}{l}16.6 \pm \\
0.25\end{array}$ & $\begin{array}{l}3.95 \pm \\
2.7\end{array}$ & $\begin{array}{l}174.1 \pm \\
57.3\end{array}$ & $\begin{array}{l}553.1 \pm \\
190\end{array}$ & 50 & $\begin{array}{l}73.1 \pm \\
21.9\end{array}$ \\
\hline & $p$ & & ns & ns & ns & ns & ns & ns & ns & ns & ns \\
\hline
\end{tabular}


Assessment of Time Delay Threshold Values till Primary Percutaneous Coronary Intervention and their Effects on Coronary Artery Flow, Myocardial Perfusion and Left Ventricular Systolic Function

\begin{tabular}{|c|c|c|c|c|c|c|c|c|c|c|c|}
\hline \multirow[t]{12}{*}{$\begin{array}{l}\text { FMC to } \\
\text { PCI }\end{array}$} & $<=45$ & $24 \%$ & $\begin{array}{l}3.9 \pm \\
5.2\end{array}$ & $\begin{array}{l}40.7 \pm \\
14.6\end{array}$ & $\begin{array}{l}12.2 \pm \\
14.35\end{array}$ & $\begin{array}{l}13.4 \pm \\
4.5\end{array}$ & $2.8 \pm 2.5$ & $\begin{array}{l}185.5 \pm \\
84.9\end{array}$ & $\begin{array}{l}627.5 \pm \\
275.8\end{array}$ & 68 & $\begin{array}{l}70.8 \pm \\
11.8\end{array}$ \\
\hline & $>45$ & $76 \%$ & $\begin{array}{l}2.8 \pm \\
0.38\end{array}$ & $\begin{array}{l}38.7 \pm \\
13.1\end{array}$ & $\begin{array}{l}16.49 \pm \\
7\end{array}$ & $\begin{array}{l}13.6 \pm \\
4.4\end{array}$ & $3.9 \pm 2.4$ & $\begin{array}{l}214 \pm \\
85.4\end{array}$ & $\begin{array}{l}676.3 \pm \\
291.5\end{array}$ & 61.1 & $\begin{array}{l}71.1 \pm \\
13.8\end{array}$ \\
\hline & $\mathbf{p}$ & & ns & ns & ns & ns & ns & ns & ns & ns & ns \\
\hline & $<=90$ & $63.5 \%$ & $\begin{array}{l}3.3 \pm \\
3.6\end{array}$ & $\begin{array}{l}38.6 \pm \\
13\end{array}$ & $\begin{array}{l}13.95 \pm \\
11.2\end{array}$ & $\begin{array}{l}13.14 \pm \\
4.03\end{array}$ & $\begin{array}{lll}3.0 & 6 \pm \\
2.05 & & \end{array}$ & $\begin{array}{l}206.4 \pm \\
89.4\end{array}$ & $\begin{array}{l}667 \pm \\
299\end{array}$ & 68.1 & $\begin{array}{l}69.9 \pm \\
10.5\end{array}$ \\
\hline & $>90$ & $36.5 \%$ & $\begin{array}{l}2.83 \pm \\
0.53 \\
\end{array}$ & $\begin{array}{l}42.8 \pm \\
15.95 \\
\end{array}$ & $\begin{array}{l}17.6 \pm \\
7.8 \\
\end{array}$ & $\begin{array}{l}14.9 \pm \\
5.6 \\
\end{array}$ & $4.8 \pm 3.2$ & \begin{tabular}{|l}
$188.5 \pm$ \\
72.5 \\
\end{tabular} & \begin{tabular}{|l|}
$618.4 \pm$ \\
231.3 \\
\end{tabular} & 50 & \begin{tabular}{|l}
$74.1 \pm$ \\
18.5 \\
\end{tabular} \\
\hline & p & & ns & ns & ns & ns & $\mathrm{ns}(0.076)$ & ns & ns & ns & ns \\
\hline & $<=135$ & $74.1 \%$ & $\begin{array}{l}3.29 \pm \\
3.3 \\
\end{array}$ & \begin{tabular}{|l|}
$39.5 \pm$ \\
14.1 \\
\end{tabular} & $\begin{array}{l}14.6 \pm \\
11 \\
\end{array}$ & \begin{tabular}{|l|}
$13.48 \pm$ \\
4.3 \\
\end{tabular} & \begin{tabular}{|l}
$3.2 \pm$ \\
2.2 \\
\end{tabular} & \begin{tabular}{|l}
$205.6 \pm$ \\
86.5 \\
\end{tabular} & \begin{tabular}{|l|l|}
$667.4 \pm$ \\
287.9 \\
\end{tabular} & 66.7 & $\begin{array}{l}70.2 \pm \\
11.3 \\
\end{array}$ \\
\hline & $>135$ & $25.9 \%$ & $\begin{array}{l}2.46 \pm \\
0.29\end{array}$ & $\begin{array}{l}39.86 \pm \\
10.1\end{array}$ & $\begin{array}{l}16.9 \pm \\
6.09\end{array}$ & $\begin{array}{l}13.8 \pm \\
5.4\end{array}$ & $\begin{array}{l}5.6 \pm \\
3.4\end{array}$ & $\begin{array}{l}173.7 \pm \\
78.95\end{array}$ & $\begin{array}{l}570.3 \pm \\
253\end{array}$ & 42.9 & $\begin{array}{l}76 \pm \\
20.3\end{array}$ \\
\hline & $\mathbf{p}$ & & ns & ns & ns & ns & ns & ns & ns & ns & ns \\
\hline & $<=180$ & $84.1 \%$ & $\begin{array}{l}3.2 \pm \\
3.17 \\
\end{array}$ & \begin{tabular}{|l|}
$39.4 \pm$ \\
13.9 \\
\end{tabular} & $\begin{array}{l}14.6 \pm \\
10.6 \\
\end{array}$ & $\begin{array}{l}13.4 \pm \\
4.4 \\
\end{array}$ & $\begin{array}{l}3.4 \pm \\
2.5 \\
\end{array}$ & \begin{tabular}{|l|}
$206.6 \pm$ \\
84.1 \\
\end{tabular} & \begin{tabular}{|l|}
$669.3 \pm$ \\
279.3 \\
\end{tabular} & 66.1 & \begin{tabular}{|l}
$70.04 \pm$ \\
11.14 \\
\end{tabular} \\
\hline & $>180$ & $15.9 \%$ & $\begin{array}{l}2.5 \pm \\
0.3 \\
\end{array}$ & $\begin{array}{l}45 \pm \\
2.8 \\
\end{array}$ & $\begin{array}{l}23 \pm \\
1.9\end{array}$ & $\begin{array}{l}16.2 \pm \\
6.6\end{array}$ & $\begin{array}{l}5.35 \pm \\
0.78\end{array}$ & $\begin{array}{l}82.5 \pm \\
19.1 \\
\end{array}$ & $\begin{array}{l}273 \pm \\
69.3 \\
\end{array}$ & 0 & $\begin{array}{l}87.7 \pm \\
30.3 \\
\end{array}$ \\
\hline & p & & ns & ns & $\begin{array}{l}2.9 \times 10^{-7} \\
<0.05\end{array}$ & ns & ns & $<0.05$ & $<0.05$ & & ns \\
\hline
\end{tabular}

Abbreviations: FMC= First Medical Contact; Time (min)= pain to FMC time (minutes); VD=Peak diastolic velocity; VS=Peak systolic velocity; TVI D= Diastolic time velocity integral; TVI S=Systolic time velocity integral; PHT=Pressure half time; DDT=Diastolic deceleration time; BPM: Beat per minute; ns= non significant.

Table 7. Myocardial biomarker maximal blood levels

\begin{tabular}{|c|c|c|c|c|}
\hline & TIME (min) & Patients(\%) & MAX.CPK [u/l] & MAX. TROPONIN[ng/ml] \\
\hline \multirow[t]{15}{*}{ Pain to PCI } & $<=90$ & $4.1 \%$ & $3068.9 \pm 2213.7$ & $77 \pm 55.5$ \\
\hline & $>90$ & $95.9 \%$ & $1477.2 \pm 1252.3$ & $50.9 \pm 45.1$ \\
\hline & p-value & & ns & ns \\
\hline & $<=120$ & $27 \%$ & $2122.2 \pm 1922$ & $58.5 \pm 47.1$ \\
\hline & $>120$ & $73 \%$ & $1464 \pm 1197$ & $51.6 \pm 46.8$ \\
\hline & p-value & & ns & ns \\
\hline & $<=180$ & $53.5 \%$ & $1664.9 \pm 1670.8$ & $55.9 \pm 44.7$ \\
\hline & $>180$ & $46.5 \%$ & $1638.5 \pm 1217$ & $51.3 \pm 49.3$ \\
\hline & P-value & & ns & ns \\
\hline & $<=230$ & $67 \%$ & $1634.7 \pm 1561$ & $50.8 \pm 44.6$ \\
\hline & $>230$ & $33 \%$ & $1689.2 \pm 1245$ & $60.03 \pm 51.5$ \\
\hline & p-value & & ns & ns \\
\hline & $<=300$ & $80.6 \%$ & $1573.9 \pm 1491$ & $49.2 \pm 45.2$ \\
\hline & $>300$ & $19.4 \%$ & $1689.2 \pm 1245$ & $84.2 \pm 43$ \\
\hline & p-value & & ns & 0.03 \\
\hline
\end{tabular}


Assessment of Time Delay Threshold Values till Primary Percutaneous Coronary Intervention and their Effects on Coronary Artery Flow, Myocardial Perfusion and Left Ventricular Systolic Function

\begin{tabular}{|c|c|c|c|c|}
\hline \multirow[t]{12}{*}{ Pain to FMC } & $<=60$ & $45 \%$ & $1969.7 \pm 1829.6$ & $52 \pm 48.6$ \\
\hline & $>60$ & $55 \%$ & $1550.8 \pm 1322.3$ & $54.3 \pm 46.5$ \\
\hline & p-value & & ns & ns \\
\hline & $<=90$ & $68 \%$ & $1720.5 \pm 1635.7$ & $50.8 \pm 45.9$ \\
\hline & $>90$ & $32 \%$ & $1588.1 \pm 1269.5$ & $56.6 \pm 47.8$ \\
\hline & p-value & & ns & ns \\
\hline & $<=120$ & $73 \%$ & $1693.6 \pm 1568.7$ & $55.8 \pm 47.7$ \\
\hline & $>120$ & $27 \%$ & $1571 \pm 1246.8$ & $49.4 \pm 49.5$ \\
\hline & p-value & & ns & ns \\
\hline & $<=180$ & $88 \%$ & $1598.4 \pm 1471.8$ & $52.1 \pm 47.7$ \\
\hline & $>180$ & $12 \%$ & $2005.8 \pm 1395.6$ & $64.4 \pm 39.8$ \\
\hline & p-value & & ns & ns \\
\hline \multirow[t]{12}{*}{ FMC to PCI } & $<=45$ & $24 \%$ & $1849.5 \pm 1654.4$ & $52.4 \pm 43.5$ \\
\hline & $>45$ & $76 \%$ & $1534.3 \pm 1334.2$ & $54.6 \pm 49$ \\
\hline & p-value & & ns & ns \\
\hline & $<=90$ & $63.5 \%$ & $1725.3 \pm 1591$ & $49.9 \pm 43.8$ \\
\hline & $>90$ & $36.5 \%$ & $1422.5 \pm 937.2$ & $65.9 \pm 54.6$ \\
\hline & p-value & & ns & ns \\
\hline & $<=135$ & $74.1 \%$ & $1689.6 \pm 1517.2$ & $53.7 \pm 44.9$ \\
\hline & $>135$ & $25.9 \%$ & $1379.2 \pm 967.2$ & $53.8 \pm 61.3$ \\
\hline & p-value & & ns & ns \\
\hline & $<=180$ & $84.1 \%$ & $1620.5 \pm 1471.2$ & $53.2 \pm 56.7$ \\
\hline & $>180$ & $15.9 \%$ & $2579.7 \pm 760.3$ & $68.3 \pm 54.9$ \\
\hline & p-value & & ns & ns \\
\hline
\end{tabular}

Abbreviations: $\mathrm{n}=$ number of patients; $\mathrm{FMC}=$ First Medical Contact; Time (min)= pain to FMC time (minutes); Max. Trop=Maximal Troponin level; Max. CPK=Maximal Creatine phosphokinase level; ns= non significant.

Table 8. Left ventricular systolic function

\begin{tabular}{|c|c|c|c|c|c|c|c|c|c|c|c|}
\hline & $\begin{array}{l}\text { T I M E } \\
\text { (min) }\end{array}$ & $\begin{array}{l}\text { Patients } \\
(\%)\end{array}$ & $\begin{array}{l}\text { L V E F - } \\
\text { EARLY } \\
(\%) \\
\end{array}$ & \begin{tabular}{|l|} 
L V E F - \\
LATE \\
$(\%)$
\end{tabular} & $\begin{array}{l}\text { DLVEF } \\
(\%)\end{array}$ & $\begin{array}{l}\text { LV WMSI } \\
\text { EARLY }\end{array}$ & \begin{tabular}{|l|} 
LV WMSI \\
LATE
\end{tabular} & $\begin{array}{lr}\Delta & \text { LV } \\
\text { WMSI }\end{array}$ & 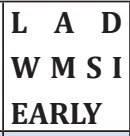 & $\begin{array}{ll}\text { L A D } \\
\text { W } & \text { M S I } \\
\text { LATE } \\
\end{array}$ & $\begin{array}{l}\Delta \quad \text { LAD } \\
\text { WMSI }\end{array}$ \\
\hline \multirow[t]{11}{*}{$\begin{array}{l}\text { Pain to } \\
\text { PCI }\end{array}$} & $<=90$ & $4.1 \%$ & $\begin{array}{l}34.6 \pm \\
4.4\end{array}$ & $\begin{array}{ll}41.3 & \pm \\
5.6 & \end{array}$ & $6.7 \pm 4.8$ & $\begin{array}{ll}1.64 & \pm \\
0.27 & \end{array}$ & $\begin{array}{ll}1.82 & \pm \\
0.46 & \end{array}$ & $\begin{array}{l}0.16 \pm \\
0.34\end{array}$ & $\begin{array}{ll}1.99 & \pm \\
0.41 & \end{array}$ & $\begin{array}{ll}2.13 & \pm \\
0.31 & \end{array}$ & $\begin{array}{l}0.16 \pm \\
0.84\end{array}$ \\
\hline & $>90$ & $95.9 \%$ & $\begin{array}{l}36.9 \pm \\
6.6\end{array}$ & $\begin{array}{ll}45 & \pm \\
9.1 & \end{array}$ & $8 \pm 8.1$ & $1.6 \pm 0.26$ & \begin{tabular}{ll|}
1.52 & \pm \\
0.35 & \\
\end{tabular} & $\begin{array}{l}-0.12 \pm \\
0.31\end{array}$ & $\begin{array}{l}1.94 \\
0.49\end{array}$ & $\begin{array}{ll}1.73 & \pm \\
0.51 & \end{array}$ & $\begin{array}{l}-0.27 \pm \\
0.45\end{array}$ \\
\hline & p & & ns & ns & ns & ns & ns & ns & ns & ns & ns \\
\hline & $<=120$ & $27 \%$ & $\begin{array}{ll}33.8 \quad \pm \\
5.3\end{array}$ & $\begin{array}{ll}44.7 & \pm \\
7.9 & \\
\end{array}$ & $10.3 \pm 8.8$ & $\begin{array}{l}1.69 \\
0.27 \\
\end{array}$ & $\begin{array}{l}1.64 \\
0.36 \\
\end{array}$ & $\begin{array}{l}-0.05 \pm \\
0.34\end{array}$ & $\begin{array}{ll}2.09 & \pm \\
0.5 & \end{array}$ & $\begin{array}{ll}1.91 & \pm \\
0.51 & \end{array}$ & $\begin{array}{l}-0.15 \pm \\
0.57\end{array}$ \\
\hline & $>120$ & $73 \%$ & $\begin{array}{l}37.9 \pm \\
6.5\end{array}$ & $\begin{array}{ll}44.5 & \pm \\
9.3 & \\
\end{array}$ & $6.8 \pm 7.2$ & $\begin{array}{l}1.58 \\
0.27\end{array}$ & $\begin{array}{ll}1.52 & \pm \\
0.36 & \\
\end{array}$ & $\begin{array}{l}-0.11 \pm \\
0.31\end{array}$ & $\begin{array}{ll}1.9 & \pm \\
0.5 & \\
\end{array}$ & $\begin{array}{ll}1.72 \pm \\
0.5\end{array}$ & $\begin{array}{l}-0.26 \pm \\
0.42 \\
\end{array}$ \\
\hline & $\mathbf{P}$ & & $\begin{array}{l}0.0022 \\
<0.05\end{array}$ & ns & ns & ns & ns & ns & ns & ns & ns \\
\hline & $<=180$ & $53.5 \%$ & $\begin{array}{ll}36.2 \quad \pm \\
6.7 & \\
\end{array}$ & $\begin{array}{l}46.3 \pm \\
8.4\end{array}$ & $10.1 \pm 8.8$ & $1.6 \pm 0.26$ & $\begin{array}{|ll|}1.57 & \pm \\
0.33 & \\
\end{array}$ & $\begin{array}{l}-0.08 \pm \\
0.35 \\
\end{array}$ & $\begin{array}{ll}1.98 & \pm \\
0.5 & \\
\end{array}$ & $\begin{array}{ll}1.85 & \pm \\
0.46 & \\
\end{array}$ & $\begin{array}{l}-0.21 \pm \\
0.58 \\
\end{array}$ \\
\hline & $>180$ & $46.5 \%$ & $\begin{array}{l}37.1 \pm \\
6.1\end{array}$ & $\begin{array}{ll}42.7 & \pm \\
9 & \\
\end{array}$ & $5.5 \pm 5.8$ & $\begin{array}{l}1.61 \\
0.27\end{array}$ & $\begin{array}{l}1.53 \\
0.39\end{array}$ & $\begin{array}{l}-0.1 \pm \\
0.3\end{array}$ & $\begin{array}{ll}1.92 & \pm \\
0.47 & \\
\end{array}$ & $\begin{array}{l}1.69 \pm \\
0.54\end{array}$ & $\begin{array}{l}-0.25 \pm \\
0.35\end{array}$ \\
\hline & p & & ns & 0.054 & $\begin{array}{l}0.005 \\
<0.05\end{array}$ & ns & ns & ns & ns & ns & ns \\
\hline & $<=230$ & $67 \%$ & \begin{tabular}{|l|}
36.8 \\
6.7 \\
\end{tabular} & $\begin{array}{|ll|}45.9 & \pm \\
8.7 & \\
\end{array}$ & $9.1 \pm 8.3$ & \begin{tabular}{|l|}
1.57 \\
0.25 \\
\end{tabular} & $\begin{array}{l}1.49 \\
0.33 \\
\end{array}$ & $\begin{array}{l}-0.12 \pm \\
0.32\end{array}$ & $\begin{array}{ll}1.93 & \pm \\
0.48 & \\
\end{array}$ & $\begin{array}{ll}1.73 & \pm \\
0.48 & \\
\end{array}$ & $\begin{array}{l}-0.27 \pm \\
0.53\end{array}$ \\
\hline & $>230$ & $33 \%$ & $\begin{array}{ll}36.4 \quad \pm \\
5.8\end{array}$ & $\begin{array}{ll}41.8 & \pm \\
8.6 & \end{array}$ & $5.3 \pm 5.8$ & \begin{tabular}{|l|}
1.67 \\
0.27 \\
\end{tabular} & $\begin{array}{ll}1.64 & \pm \\
0.41 & \\
\end{array}$ & $\begin{array}{l}-0.05 \pm \\
0.31\end{array}$ & $\begin{array}{ll}1.98 & \pm \\
0.49 & \\
\end{array}$ & $\begin{array}{ll}1.81 & \pm \\
0.55 & \\
\end{array}$ & $\begin{array}{l}-0.18 \pm \\
0.33 \\
\end{array}$ \\
\hline
\end{tabular}


Assessment of Time Delay Threshold Values till Primary Percutaneous Coronary Intervention and their Effects on Coronary Artery Flow, Myocardial Perfusion and Left Ventricular Systolic Function

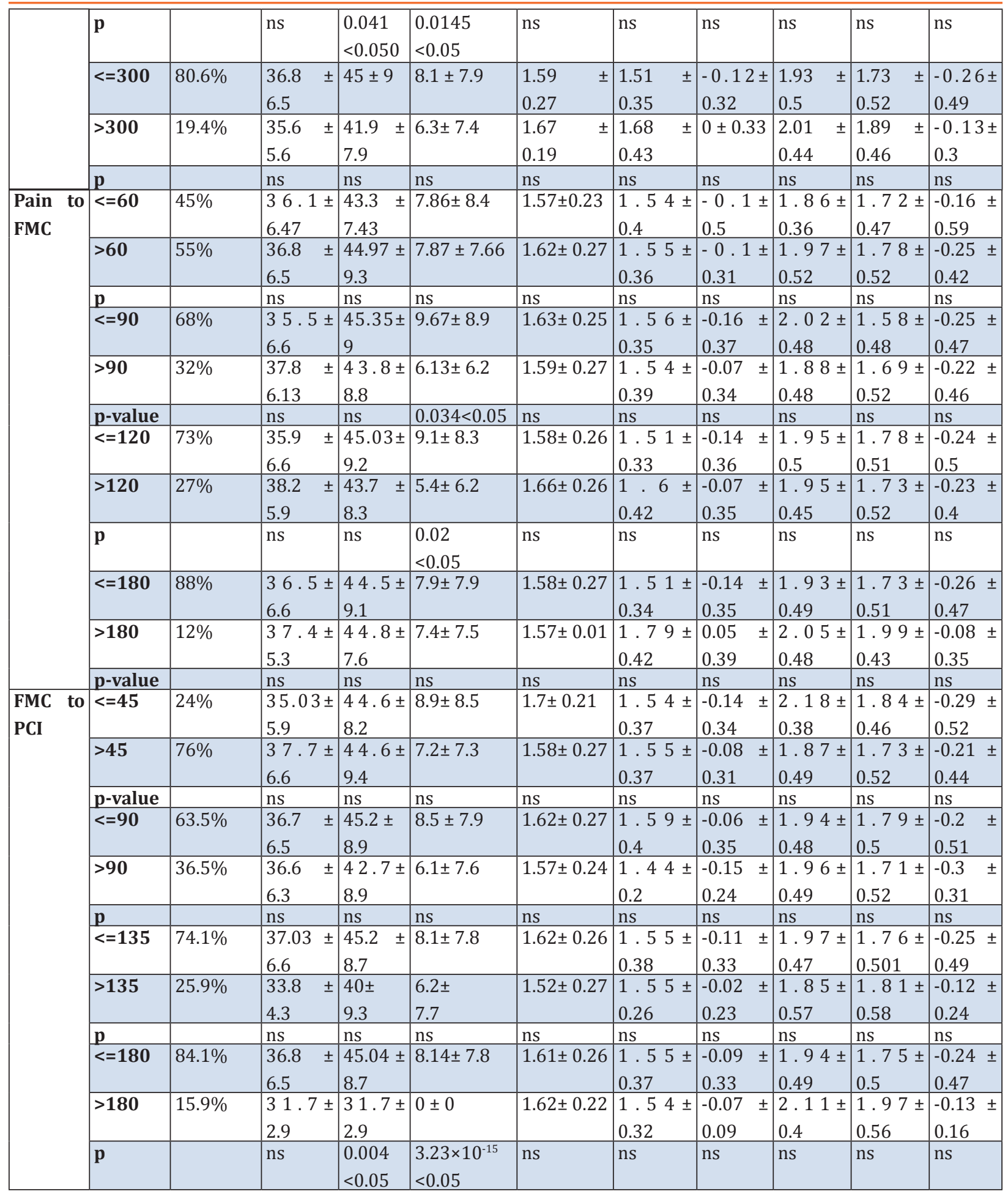

Abbreviations: FMC= First Medical Contact; Time (min)= pain to FMC time (minutes); LVEF-Early= Early left ventricular ejection fraction; LVEF-Late= Late left ventricular ejection fraction; $\triangle \mathrm{LVEF}=$ (LVEF-Late) - (LVEF-Early); LV WMSI EARLY= Early left ventricular wall motion index; LV WMSI LATE= Late left ventricular wall motion index; $\triangle \mathrm{LV}$ WMSI=(LV WMSI LATE) - (LV WMSI EARLY); LAD WMSI EARLY= Early wall motion score index of segments supplied by the left anterior descending coronary artery; LAD WMSI LATE= Late wall motion score index of segments supplied by the left anterior descending coronary artery; $\triangle$ LAD WMSI= (LAD WMSI LATE) - (LAD WMSI EARLY); ns= non significant. 
Assessment of Time Delay Threshold Values till Primary Percutaneous Coronary Intervention and their Effects on Coronary Artery Flow, Myocardial Perfusion and Left Ventricular Systolic Function

\section{DiscusSion}

In the present, study, the effects of time delays from onset of chest pain till primary PCI on infract related artery coronary flow, myocardial perfusion parameters, myocardial injury and recovery of LV systolic function were evaluated. Shorter intervals of pain to PCI were associated with better angiographic infarct related coronary artery flow and myocardial perfusion, less myocardial damage, and larger improvement in $\mathrm{LV}$ systolic function. In addition, shorter pain to FMC, and shorter FMC to PCI intervals were associated with larger improvement in LV systolic function.

In the current study, the recently recommended terminology of time intervals till PCI (2017 European guidelines for the management of patients presenting with acute STEMI) was adopted (18). Thus chest pain to FMC, FMC to PCI and pain to PCI time intervals were evaluated according to the new guidelines.

It is important to mention that the patient factor, chest pain to FMC time intervals, did not change over the years; however the medical system factor, FMC to PCI and consequently pain to PCI time intervals decreased significantly after 2010. The more recent European society of cardiology guidelines (18) recommend to keep the interval between STEMI diagnosis to PCI less than $120 \mathrm{~min}$, and in primary PCI hospitals less than $60 \mathrm{~min}$; while the previous recommendations from 2014 (20), suggest to keep door to balloon time intervals less than 60 minutes and pain to PCI times less than 2-3hours (20).

The hypothesis of the present study that time delays from the onset of chest pain till primary PCI affect both the coronary flow and myocardial perfusion in the infarct related coronary artery tended to reach statistical significance. While shorter pain to PCI intervals tended to be associated with better angiographic outcome, pain to FMC and FMC to PCI time intervals did not affect the angiographic results. In concordance with the findings of the present study, prevalence of slow flow in the infarct related artery in patients with STEMI undergoing primary PCI was reported to be higher when the pain to balloon time was larger than 4 hours (21). Moreover it was reported that only the combination of a short ( $<90 \mathrm{~min}$ ) door to balloon time and a short chest pain-to-door time was associated with the improvement of clinical outcome
$(22,23)$. Previously a threshold value for door to balloon time was examined (i.e., $\leq 30$ vs $>30 \mathrm{~min}, \leq 45$ vs $>45 \mathrm{~min}$, and $\leq 60 \mathrm{vs}>60 \mathrm{~min}$ ) and surprisingly, the prevalence of final TIMI flow less than or equal to 2 was not affected by these time intervals(24).

Concerning the function of coronary microcirculation, shorter pain to $\mathrm{PCI}$ intervals were associated with better ST-elevation resolution, while shorter FMC to PCI time intervals had more partial ST-elevation resolution. Since the function of the coronary microcirculation and coronary artery velocities are dynamic and changing during the course of hospitalization after PPCI (17), LAD velocity parameters were evaluated early after primary PCI and before discharge from the hospital. A trend towards lower LAD velocities in patients with shorter time intervals was noticed, possibly related to a trend of larger LAD diameters in these patients.

Regarding myocardial injury; shorter pain to PCI time intervals (less than $300 \mathrm{~min}$ ) was associated with lower maximal troponin blood levels. As a result, the increase in LVEF was larger in patients with shorter pain to PCI intervals (less than 180 and $230 \mathrm{~min}$ ). In addition, shorter pain to FMC and FMC to PCI intervals, were associated with large improvement in LV systolic function. Similar to the findings of the present study, it was reported that shortening of the duration between chest pain onset and reperfusion to less than 4.0 hours was critical in reducing myocardial necrosis and improving heart function and 30-day mortality (23). Ultrashort door to balloon time $(<45 \mathrm{~min})$ did not improve left ventricular systolic function (23), and did not improve mortality $(24,25)$.

Shortening of door to balloon time has been verified to benefit patients presenting early and/or with high risk in terms of reducing mortality $(23,26-$ 28). The emphasis regarding time is on "presenting early". As already mentioned, in the present study, the patient factor-pain to FMC- did not change overtime, while the medical system factor-FMC to PCI time did shorten and lead to reduction in the ischemic time from chest pain till primary PCI. More importantly, further shortening of FMC to PCI time did not improve outcome $(24,29)$. These findings are in parallel with the patho-physiology of the time course of development of myocardial injury in acute STEMI. Following the abrupt thrombotic occlusion of 
Assessment of Time Delay Threshold Values till Primary Percutaneous Coronary Intervention and their Effects on Coronary Artery Flow, Myocardial Perfusion and Left Ventricular Systolic Function

an epicardial coronary artery, a wave front of necrosis begins from the subendocardial necrotic area to the periphery and towards the epicardium (6). Thus, time is muscle, and the reopening of the occluded vessel should be performed as fast as possible. Pain to PCI interval should be as short as possible; however, if pain to FMC is so long, shortening FMC to PCI interval will not yield significant improvement in outcome. Therefore, future efforts to reduce pain to FMC time rather than achieving ultra-short FMC to PCI time intervals seems warranted in order to achieve better primary PCI outcomes.

\section{LIMITATIONS}

This was a single center, small study without long term follow up. Apparently, large long term multicenter studies and registries are needed.

\section{CONCLUSION}

In conclusion, decreasing pain to primary PCI time intervals improves myocardial perfusion and thus, improves LVEF. Since pain to FMC time intervals did not change overtime, reduction of total ischemic time by decreasing pain to FMC time intervals seems to be more effective than achieving ultra-short FMC to PCI time intervals.

\section{REFERENCES}

[1] Grines CL, Brown KF, Marco J, Rothbaum D, Stone GW, O'Keefe J, et al. A comparison of immediate angioplasty with thrombolytic therapy for acute myocardial infarction: the Primary Angioplasty in Myocardial Infarction Study Group. N Engl J Med. 1993; 328:673-679.

[2] Zijlstra F, de Boer MJ,HoorntjeJC, Reiffers S, Reiber J, Suryapranata H. A comparison of immediate angioplasty with intravenous streptokinase in acute myocardial infarction. N Eng J Med 1993; 38:680-684.

[3] GUSTO IIb investigators. A clinical trial comparing primary angioplasty with tissue plasminogen activator for acute myocardial infarction. N Eng J Med 1997; 336:1621-1628.

[4] De Luca G, van 't Hof AW, de Boer MJ, Ottervanger JP, Hoorntje JC, Gosselink AT, et al. Time to treatment significantly affects the extent of STsegement resolution and myocardial blush in patients with acute myocardial infarction treated by primary angioplasty. European Heart Journal 2004; 25:1009-1013.

[5] Kushner FG, Hand M, Smith SC, Jr King SB 3rd, Anderson JL, Antman EM, et al. 2009 focused updates: ACC/AHA guidelines for the management of patients with ST-elevation myocardial infarction (updating the 2004 guideline and 2007 focused update) and ACC/ AHA/SCAI guidelines on percutaneous coronary intervention (updating the 2005 guideline and 2007 focused update) a report of the American College of Cardiology Foundation/American Heart Association Task Force on Practice Guidelines. J Am Coll Cardiol. 2009; 54:22052241.

[6] Reimer KA, Lowe JE, Rasmussen MM, Jennings RB. The wavefront of ischemic cell death. 1. Myocardial infarct size vs duration of coronary occlusion in dogs. Circulation 1977; 56:786-794.

[7] Nallamothu BK, Bradley EH, Krumholz HM. Time to treatment in primary percutaneous coronary intervention. N Engl J Med. 2007; 357:16311638.

[8] Cannon CP, Gibson CM, Lambrew CT, et al. Relationship of symptom-onset-to-balloon time and door-to-balloon time with mortality in patients undergoing angioplasty for acute myocardial infarction. JAMA. 2000; 283:29412947.

[9] McNamara RL, Wang Y, Herrin J, Curtis JP, Bradley $\mathrm{EH}$, Magid DJ, et al. NRMI Investigators. Effect of door-to-balloon time on mortality in patients with ST-segment elevation myocardial infarction. J Am Coll Cardiol. 2006; 47:2180-2186.

[10] van de Werf F, Ardissino D, Betriu A, Cokkinos DV, Falk E, Fox KA, et al. Task Force on the Management of Acute Myocardial Infarction of the European Society of Cardiology. Management of acute myocardial infarction in patients presenting with ST-segment elevation. The Task Force on the Management of Acute Myocardial Infarction of the European Society of Cardiology. Eur Heart J. 2003; 24:28-66. 
Assessment of Time Delay Threshold Values till Primary Percutaneous Coronary Intervention and their Effects on Coronary Artery Flow, Myocardial Perfusion and Left Ventricular Systolic Function

[11] Antman EM, Anbe DT, Armstrong PW, Bates ER, Green LA, Hand M, et al. ACC/AHA guidelines for the management of patients with ST-elevation myocardial infarction: a report of the American college of cardiology/American heart association task force of practice guidelines. J Am Coll Cardiol. 2004; 44: el-211.

[12] European Society of Cardiology. Guidelines on myocardial revascularization. Eur Heart J 2010; 31:2501-2555.

[13] Centurión OA. The open artery hypothesis: beneficial effects and long-term prognostic importance of patency of the infarct-related coronary artery. Angiology. 2007; 58:34-44.

[14] Eldrin L, Lemuel M, Jean R, Sacks FM, Arnold JM, Warnica JW, et al. Predictors of late development of heart failure in stable survivors of myocardial infarction. J Am Coll Cardiol. 2003; 42:1446-1453.

[15] Gibson CM, Cannon CP, Murphy SA, Ryan KA, Mesley R, Marble SJ, et al. Relationship of TIMI Myocardial Perfusion Grade to Mortality After Administration of Thrombolytic Drugs. Circulation. 2000; 101: 125-130.

[16] Stone GW, Peterson MA, Lansky AJ, Dangas G, Mehran R, Leon MB. Impact of normalized myocardial perfusion after successful angioplasty in acute myocardial infarction, J Am Coll Cardiol. 2002; 39: 591-597.

[17] Sharif D, Rofe G, Sharif-Rasslan A, Goldhammer E, Makhoul N, Shefer A, et al. Analysis of serial coronary artery flow patterns early after primary angioplasty: new insights into the dynamics of the microcirculation. Isr Med Assoc J. 2008; 10: 440-4.

[18] Ibanez B, James S, Agewall S, Antunes MJ, Bucciarelli-Ducci C, Bueno H, et al. 2017 ESC Guidelines for the management of acute myocardial infarction in patients presenting with ST-segment elevation - Web Addenda. European Heart Journal. 2017, 00, 1-66.

[19] van't Hof AW, Liem A, Suryapranata H, Hoorntje JC, de Boer MJ, Zijlstra F. Angiographic assessment of myocardial reperfusion in patients treated with primary angioplasty for acute myocardial infarction: myocardial blush grade. Zwolle Myocardial Infarction Study Group. Circulation. 1998; 97: 2302-2306.

[20] Windecker S, Kolh P, Alfonso F, Collet JP, Cremer J, Falk V, et al. 2014 ESC/EACTS Guidelines on myocardial revascularization. The Task Force on Myocardial Revascularization of the European Society of Cardiology (ESC) and the European Association for Cardio-Thoracic Surgery (EACTS) Developed with the special contribution of the European Association of Percutaneous Cardiovascular Interventions (EAPCI). European Heart Journal, 2014, doi:10.1093/eurheartj/ ehu278, PP: 2-100.

[21] Yip HK, Chen MC, Chang HW, Hang CL, Hsieh YK, Fang CY, et al. Angiographic morphologic features of infarct-related arteries and timely reperfusion in acute myocardial infarction: Predictors of slow-flow and no-reflow phenomenon. Chest 2002; 122:1322-1332.

[22] Hannan EL, Zhong Y, Jacobs AK, Holmes DR, Walford G, Venditti FJ, et al. Effect of onsetto-door time and door-to-balloon time on mortality in patients undergoing percutaneous coronary interventions for ST-segment elevation myocardial infarction. Am J Cardiol. 2010; 106:143-147.

[23] Brodie BR, Gersh BJ, Stuckey T, Witzenbichler B, Guagliumi G, Peruga JZ, et al. When is doorto-balloon time critical? Analysis from the HORIZONS-AMI (Harmonizing Outcomes with Revascularization and Stents in Acute Myocardial Infarction) and CADILLAC (Controlled Abciximab and Device Investigation to Lower Late Angioplasty Complications) trials. J Am Coll Cardiol. 2010; 56:407-413.

[24] Ho YC, Tsai TH, Sung PH, Chen YL, Chung SY, Yang $\mathrm{CH}$, et al. Minimizing door-to-balloon time is not the most critical factor in improving clinical outcome of ST-elevation myocardial infarction patients undergoing primary percutaneous. Coronary Intervention Crit Care Med 2014; 42:1788-1796.

[25] Menees DS, Peterson ED, Wang Y, Curtis JP, Messenger JC, Rumsfeld JS, et al. Door-toballoon time and mortality among patients 
Assessment of Time Delay Threshold Values till Primary Percutaneous Coronary Intervention and their Effects on Coronary Artery Flow, Myocardial Perfusion and Left Ventricular Systolic Function

undergoing primary PCI. N Engl J Med. 2013; 369:901-909.

[26] Shiomi H, Nakagawa Y, Morimoto T, Furukawa Y, NakanoA, Shirai S, et al. Association of onset to balloon and door to balloon time with long term clinical outcome in patients with ST elevation acute myocardial infarction having primary percutaneous coronary intervention: observational study. BMJ 2012; 344:e3257.

[27] Brodie BR, Hansen C, Stuckey TD, Richter S, Versteeg DS, Gupta N, et al. Door-to-balloon time with primary percutaneous coronary intervention for acute myocardial infarction impacts late cardiac mortality in high-risk patients and patients presenting early after the onset of symptoms. J AmColl Cardiol. 2006; 47:289-95.

[28] Kong PK, Connolly D, Varma C, Lip G, Millane T, Davis R, et al. High-risk myocardial infarction patients appear to derive more mortality benefit from short door-to-balloon time than low-risk patients. Int J Clin Pract 2009; 63:1693-701.

[29] Lai CH, Chang KC, Liao PC, Wu CT, Lai WT, Wu CJ, et al. Effects of door-to-balloon times on outcomes in Taiwanese patients receiving primary percutaneous coronary intervention: A report of Taiwan acute coronary syndrome full spectrum registry. Acta Cardiol Sin 2015; 31:215_225

Citation: Dawod Sharif, Mohamad Watad, Yasmine Sharif, Amal Sharif-Rasslan, Uri Rosenschein. Assessment of Time Delay Threshold Values till Primary Percutaneous Coronary Intervention and their Effects on Coronary Artery Flow, Myocardial Perfusion and Left Ventricular Systolic Function. Archives of Emergency Medicine and Intensive Care. 2018; 1(2): 07-21.

Copyright: (C) 2018 Dawod Sharif, Mohamad Watad, Yasmine Sharif, Amal Sharif-Rasslan, Uri Rosenschein. This is an open access article distributed under the Creative Commons Attribution License, which permits unrestricted use, distribution, and reproduction in any medium, provided the original work is properly cited. 